\title{
Thermal regime of the Costa Rican convergent margin: 1. Along-strike variations in heat flow from probe measurements and estimated from bottom-simulating reflectors
}

\section{Robert N. Harris}

College of Oceanic and Atmospheric Sciences, Oregon State University, Corvallis, Oregon 97331, USA

(rharris@coas.oregonstate.edu)

\section{Ingo Grevemeyer}

Leibniz Institute of Marine Sciences at University of Kiel (IFM-GEOMAR), D-24148 Kiel, Germany

\section{César R. Ranero}

Barcelona Center for Subsurface Imaging, ICREA, Instituto de Ciencias del Mar, CSIC, Pg. Maritim de la Barcelona 37-49, E-08003 Barcelona, Spain

\section{Heinrich Villinger}

Department of Geosciences, University of Bremen, PO Box 330 440, D-28334 Bremen, Germany

\section{Udo Barckhausen}

Federal Institute for Geosciences and Natural Resources, Stilleweg 2, D-30655 Hannover, Germany

\section{Thomas Henke}

Federal Institute for Geosciences and Natural Resources, Stilleweg 2, D-30655 Hannover, Germany

Now at TEEC, Burgwedeler Str. 89, D-30916 Isernhagen, Germany

\section{Christian Mueller}

Federal Institute for Geosciences and Natural Resources, Stilleweg 2, D-30655 Hannover, Germany

\author{
Soenke Neben \\ Federal Institute for Geosciences and Natural Resources, Stilleweg 2, D-30655 Hannover, Germany \\ Deceased
}

[1] The thermal structure of convergent margins provides information related to the tectonics, geodynamics, metamorphism, and fluid flow of active plate boundaries. We report 176 heat flow measurements made with a violin bow style probe across the Costa Rican margin at the Middle America Trench. The probe measurements are collocated with seismic reflection lines. These seismic reflection lines show widespread distribution of bottom-simulating reflectors (BSRs). To extend the spatial coverage of heat flow measurements we estimate heat flow from the depth of BSRs. Comparisons between probe measurements and BSR-derived estimates of heat flow are generally within $10 \%$ and improve with distance landward of the deformation front. Together, these determinations provide new information on the thermal regime of this margin. Consistent with previous studies, the margin associated with the northern Nicoya Peninsula is remarkably cool. We define better the southern boundary of the cool region. The northern extent 
of the cool region remains poorly determined. A regional trend of decreasing heat flow landward of the deformation front is apparent, consistent with the downward advection of heat by the subducting Cocos Plate. High wave number variability at a scale of $5-10 \mathrm{~km}$ is significantly greater than the measurement uncertainty and is greater south of the northern Nicoya Peninsula. These heat flow anomalies vary between approximately 20 and $60 \mathrm{~mW} \mathrm{~m}^{-2}$ and are most likely due to localized fluid flow through mounds and faults on the margin. Simple one-dimensional models show that these anomalies are consistent with flow rates of $7-15 \mathrm{~mm} \mathrm{yr}^{-1}$. Across the margin toe variability is significant and likely due to fluid flow through deformation structures associated with the frontal sedimentary prism.

Components: 11,300 words, 9 figures, 1 table.

Keywords: subduction zones; heat flow; fluid flow; Middle America Trench.

Index Terms: 3060 Marine Geology and Geophysics: Subduction zone processes (1031, 3613, 8170, 8413); 3015 Marine Geology and Geophysics: Heat flow (benthic); 3004 Marine Geology and Geophysics: Gas and hydrate systems.

Received 22 June 2010; Revised 6 October 2010; Accepted 11 October 2010; Published 15 December 2010.

Harris, R. N., I. Grevemeyer, C. R. Ranero, H. Villinger, U. Barckhausen, T. Henke, C. Mueller, and S. Neben (2010), Thermal regime of the Costa Rican convergent margin: 1. Along-strike variations in heat flow from probe measurements and estimated from bottom-simulating reflectors, Geochem. Geophys. Geosyst., 11, Q12S28, doi:10.1029/2010GC003272.

Theme: Central American Subduction System

Guest Editors: G. Alvarado, K. Hoernle, and E. Silver

\section{Introduction}

[2] Determinations of heat flow yield valuable information for understanding the thermal regime of shallow subduction zones and provide context for interpreting many hydrogeologic and geodynamic processes. Hydrogeologic processes of interest include sediment compaction, pore fluid expulsion, and other processes leading to fluid migration, as well as dehydration reactions within the subducting sediment and crust. Geodynamic processes of interest include modes of deformation along the plate interface, seismicity, and volcanism. These processes are not mutually exclusive since fluid production and migration can modify stresses and influence deformation.

[3] The Costa Rica subduction zone is one of the best studied erosive margins. It has been the focus of many studies including those of the Deep Sea Drilling Project, Ocean Drilling Program, and Integrated Drilling Program [von Huene et al., 1985; Kimura et al., 1997; Morris et al., 2003], the location of a German Science Foundation funded Priority Program project, and a focus site of the U.S. Margins program. Offshore data include 2-D and 3-D seismic data [Shipley et al., 1992; Hinz et al., 1996], wideangle seismic refraction [Ye et al., 1996; Christeson et al., 1999; Walther, 2003], swath bathymetry [von Huene et al., 2000; Ranero et al., 2008], magnetic surveys [Barckhausen et al., 2001] and seafloor observations [e.g., McAdoo et al., 1996; Sahling et al., 2008].

[4] These studies show that the margin is characterized by long-term large-scale slope subsidence and associated tectonic extension characteristic of erosive margins [Ranero and von Huene, 2000; Ranero et al., 2000; Vannucchi et al., 2003]. The morphology of the margin (Figure 1) is segmented along strike with a more stable portion where relatively smooth seafloor is subducted offshore the northern Nicoya Peninsula and a more dynamic portion where rough seafloor is subducting to the south [e.g., von Huene et al., 2000]. Both the erosive nature and segmentation of the margin affects its thermal and hydrologic state. Characterizing the thermal regime of the margin provides insights into tectonic processes and the style, location, and magnitude of subsurface fluid flow.

[5] In this paper, the first of two, we report heat flow values based on probe measurements and the location of bottom-simulating reflectors (BSRs) along multiple transects spanning the Costa Rican margin. The quality and geographic extent of these thermal data provide an unprecedented opportunity to investigate along-margin variations in heat flow. The data are used to construct a heat flow map of the margin. The focus of this paper is on high- 


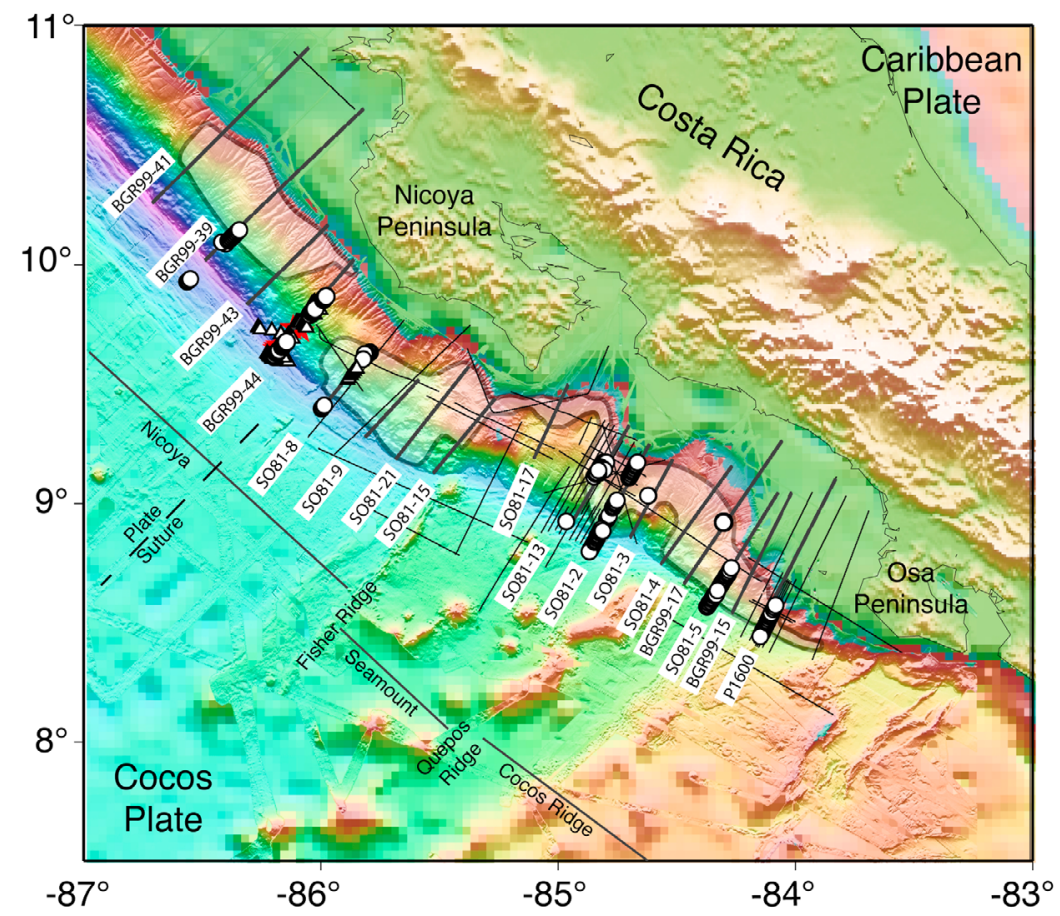

Figure 1. Map showing location of seismic lines (bold seismic lines are those analyzed in this study and by Harris et al. [2010]), heat flow data of Langseth and Silver [1996] (triangles), and new heat flow data (circles). DSDP Site 565 and ODP Sites 1039, 1040, 1041, and 1043 are shown as red stars along seismic line BGR99-44. Morphologic segments are labeled. Areas of observed bottom-simulating reflectors are outlined and shaded white. The approximate location of the plate suture is marked with a dashed line.

wave number heat flow variations that provide insight into surficial processes. Harris et al. [2010] generate finite element models to interpret lowwave number variations in heat flow and estimate temperatures along the subduction plate boundary.

\section{Regional Setting}

[6] Offshore Costa Rica the oceanic Cocos Plate subducts under the Caribbean plate forming the southern end of the Middle America Trench (Figure 1). Subduction rates vary from $70 \mathrm{~mm} / \mathrm{yr}$ offshore Guatemala to $90 \mathrm{~mm} / \mathrm{yr}$ offshore southern Costa Rica [DeMets, 2001]. The age of the Cocos Plate at the Middle America Trench decreases from $24 \mathrm{Ma}$ offshore the Nicoya Peninsula to $15 \mathrm{Ma}$ offshore the Osa Peninsula [Barckhausen et al., 2001].

[7] Seismic data and drilling offshore the Nicoya peninsula show that the margin is composed of a thick slope sediment apron, a few hundred meters to $\sim 2 \mathrm{~km}$ thick, unconformably overlying a margin of crystalline basement rock that is likely the offshore extension of igneous rocks cropping out on the Nicoya Peninsula. These low-permeability crystalline rocks are cut by numerous landward dipping normal faults. A small frontal sedimentary prism, typically $10 \mathrm{~km}$ wide or less lies seaward of the margin and is compositionally similar to the sedimentary apron [Kimura et al., 1997; Morris et al., 2003]. The incoming oceanic plate is overlain by a maximum sediment thickness of 300 $500 \mathrm{~m}$ [Kimura et al., 1997; Morris et al., 2003].

[8] The along-strike morphology of the margin is variable and largely reflects the relief of the incoming plate [von Huene et al., 2000]. We briefly review this morphology because it provides a framework for understanding the distribution of BSRs and has been linked to variations in the hydrology of the margin [Sahling et al., 2008; Ranero et al., 2008]. Three morphologically distinct segments (Figure 1) have been recognized and from north to south are termed the Nicoya, Seamount, and Cocos Ridge Segments [von Huene et al., 2004].

[9] The incoming oceanic crust within the Nicoya segment offshore southern Nicaragua and the Nicoya Peninsula has a notable tectonic history [Hey, 1977; von Huene et al., 2000; Barckhausen et al., 2001]. Oceanic crust forming the northern portion of this segment was created at the fast spreading East Pacific Rise (EPR) and oceanic 
crust to the south was formed at the slow spreading Cocos-Nazca spreading center (CNS). The boundary separating EPR from CNS crust is a combination of a triple junction trace and a fracture zone, collectively comprising a "plate suture" (Figure 1). The rough-smooth boundary from Hey [1977] is further southeast, as explained by Barckhausen et al. [2001]. Recent swath mapping north of the plate suture, however, reveals the presence of more seamounts disrupting the "smooth" sedimentary cover than previously recognized [Fisher et al., 2003]. The Nicoya segment margin is characterized by an upper slope with deep canyons, a relatively gentle middle slope with shallower canyons and recent sedimentation closer to the trench. Together these features have been interpreted to indicate relative stability of this portion of the margin compared to other segments [von Huene et al., 2000].

[10] The seamount segment lies between Fisher Ridge and Quepos Ridge south of the Nicoya segment [von Huene et al., 2000]. Here approximately $40 \%$ of the incoming seafloor is covered with seamounts having heights and diameters above the sedimentary cover of 1 to $2.5 \mathrm{~km}$ and 10 to $20 \mathrm{~km}$, respectively. The seamount segment margin is characterized by embayments and mass wasting deposits. Seismic reflection profiles image seamounts within the margin and reveal that their subduction leads to embayments, slumping, and mass wasting deposits [von Huene et al., 2000, 2004]. Rounded uplifts and domes at the surface often indicate the current position of subducted seamounts.

[11] The Cocos Ridge segment lies along the flank of the Cocos Ridge between the Quepos Plateau and the Osa Peninsula. The Cocos ridge is a bathymetric feature more than $1000 \mathrm{~km}$ long and 250 to $500 \mathrm{~km}$ wide with a bathymetric expression $2 \mathrm{~km}$ shallower than surrounding seafloor representing the trace of the Galapagos hot spot [Hey, 1977]. This region of the margin is narrow and steep and likely reflects the continuous subduction of the Cocos Ridge [von Huene et al., 2000].

\section{Seismic Data}

[12] Seismic data mainly come from cruises $R / V$ Sonne SO-81 [Hinz et al., 1996; von Huene et al., 2000; Ranero and von Huene, 2000; Ranero et al., 2007], and BGR99 [Ranero et al., 2008]. Seismic line P1600 is provided by Shell [von Huene et al., 2000]. All seismic lines were collected with large tuned air gun arrays and multichannel streamers, as described in the original papers. Seismic data were processed for signal enhancement including deconvolution and multiple attenuation, and were poststack time migrated. Selected sections are prestack depth migrated. All lines provide good imaging of the structure of the overriding plate including the sediment cover strata, BSRs and plate boundary reflections.

\section{Distribution of BSRs}

[13] BSRs were recognized in early seismic studies of the margin [Shipley et al., 1979; Yamano et al., 1982; Pecher et al., 1998; Müller et al., 2007]. Pecher et al. [1998] investigated the nature and distribution of BSRs in the study area. In general they found that BSRs are widespread in areas of margin stability, but are absent in areas characterized by slumping, except where the material failed as a coherent block. Because the BSRs are used to quantify the thermal regime across the margin, we briefly review representative seismic sections across each of the morphological segments and their implications for interpretations of heat flow.

[14] BSRs associated with the northern Nicoya segment (Figure 1) are relatively continuous along seismic lines BGR99-41, BGR99-39 (Figure 2a), and BGR99-43. With the exception of seismic line BGR99-44, BSRs first appear about $20 \mathrm{~km}$ landward of the trench and extend approximately $45 \mathrm{~km}$ landward of the trench. Along seismic line BGR99-44 BSRs only appear near the landward most extent of the line.

[15] Along the southern Nicoya segment, BSRs appear near the trench axis and extend approximately 30 to $50 \mathrm{~km}$ landward as imaged on seismic lines SO81-8, SO81-9, and SO81-21. Seismic Line SO81-21 (Figure 2b) shows a pronounced slump deposit with an overlying BSR indicating that either the mass moved as a coherent block or that the slump is old enough for the BSR to reform [von Huene et al., 2004]. In contrast, just to the south along seismic line SO81-15 (Figure 2c) the BSR is truncated by a slump headwall coincident with a subducting seamount and no BSR is observed within the slump deposit [von Huene et al., 2004].

[16] Large margin embayments characterize the Seamount segment where the subduction of seamounts causes large-scale slumping along the margin [von Huene et al., 2000]. In this segment BSRs are not observed seaward of slumps but are present landward of slump headwalls and appear 

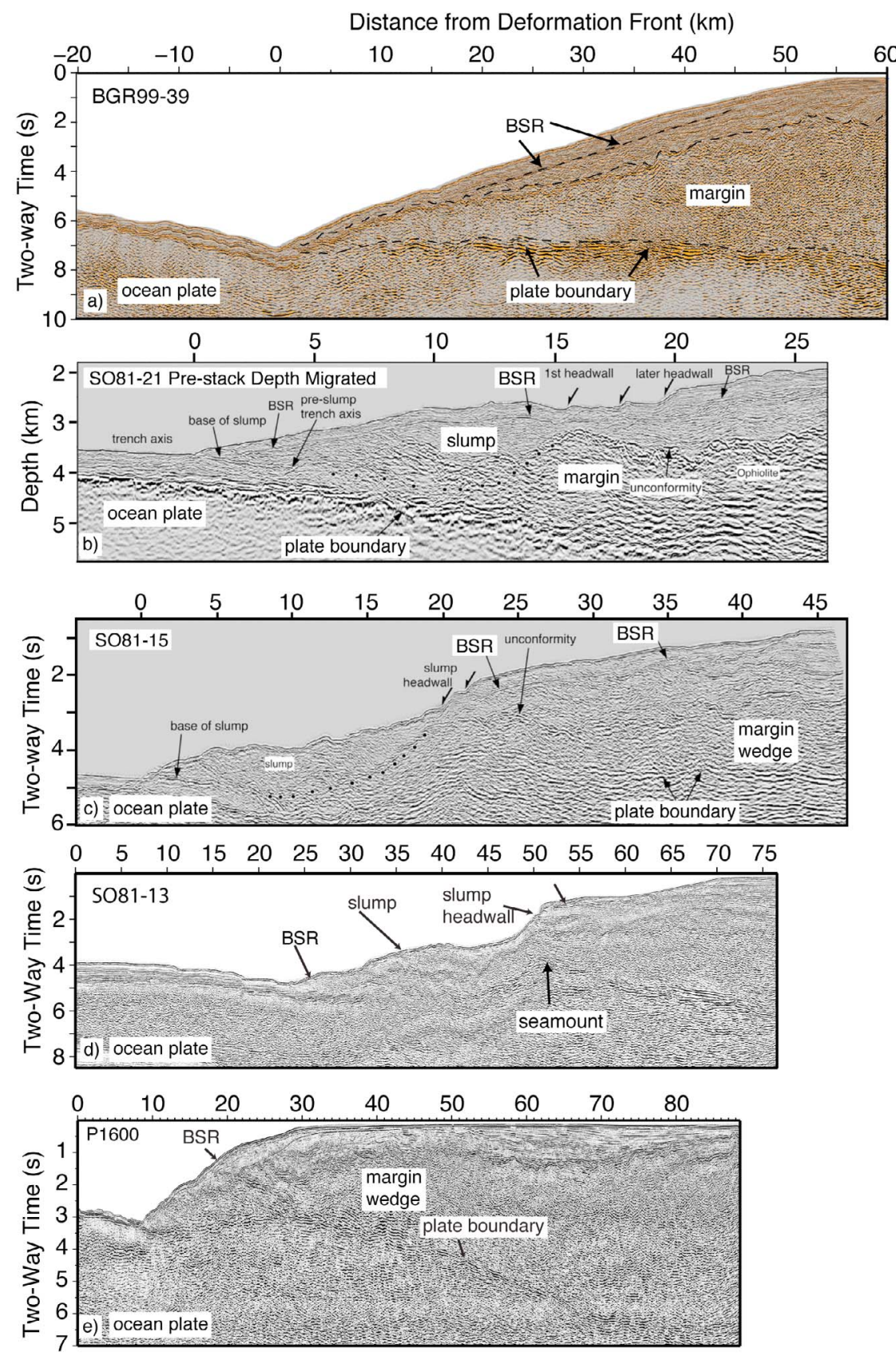

Figure 2. Poststack time migrated seismic reflection profiles across the margin, offshore Costa Rica. Seismic line locations are shown in Figure 1. Line SO81-21 is prestack depth migrated. The positions of BSRs are indicated by arrows.

truncated by them. Examples of these features can be observed on seismic lines, SO81-15 (Figure 2c) SO81-13 (Figure 2d). A rapid shallowing of BSRs toward the seafloor marks their landward extent.

[17] The Cocos Ridge segment is characterized by a steep and narrow margin slope as observed in seismic line P-1600 (Figure 2e). BSRs begin within $10 \mathrm{~km}$ of the deformation front and shallow toward the seafloor. Their landward extent decreases to the south (Figure 1).

\section{Heat Flow Transects Across the Margin}

[18] Heat flow determinations were made at 176 points (Table 1) and all come from METEOR 
Table 1. Heat Flow Data

\begin{tabular}{|c|c|c|c|c|c|c|c|c|c|c|}
\hline Profile & Penetration & $\begin{array}{l}\text { Longitude } \\
\text { (deg) }\end{array}$ & $\begin{array}{l}\text { Latitude } \\
\text { (deg) }\end{array}$ & $\begin{array}{l}\text { Water } \\
\text { Depth } \\
(\mathrm{m})\end{array}$ & $\begin{array}{c}\text { Distance }^{\mathrm{a}} \\
(\mathrm{km})\end{array}$ & $\begin{array}{l}\text { Thermal } \\
\text { Gradient } \\
\left({ }^{\circ} \mathrm{C} \mathrm{km}^{-1}\right)\end{array}$ & $\begin{array}{l}\text { Corrected } \\
\text { Thermal } \\
\text { Gradient }^{\mathrm{b}} \\
\left({ }^{\circ} \mathrm{C} \mathrm{km}^{-1}\right)\end{array}$ & $\begin{array}{c}\text { Thermal } \\
\text { Conductivity } \\
\left(\mathrm{W} \mathrm{m}^{-1} \mathrm{~K}^{-1}\right)\end{array}$ & $\begin{array}{c}\text { Heat } \\
\text { Flow }^{\mathrm{c}} \\
\left(\mathrm{mW} \mathrm{m}^{-2}\right)\end{array}$ & Instrument $^{\mathrm{d}}$ \\
\hline BGR-39 & Н0209P01 & -86.3163 & 10.3072 & 1684 & 35.7 & 45.7 & 45.1 & $0.76^{\mathrm{e}}$ & 34.3 & HP1 \\
\hline BGR-39 & Н0209P02 & -86.3140 & 10.3042 & 1676 & 35.7 & 48.0 & 46.2 & $0.76^{\mathrm{f}}$ & 35.1 & HP1 \\
\hline BGR-39 & H0209P03 & -86.3110 & 10.3013 & 1668 & 35.7 & 51.5 & 48.0 & $0.76^{\mathrm{e}}$ & 36.5 & HP1 \\
\hline BGR-39 & H0209P04 & -86.3092 & 10.2995 & 1586 & 35.7 & 54.5 & 71.8 & $0.80^{\mathrm{f}}$ & 57.4 & HP1 \\
\hline BGR-39 & Н0209P05 & -86.3080 & 10.2985 & 1535 & 35.7 & 43.8 & 72.7 & $0.82^{\mathrm{e}}$ & 59.6 & HP1 \\
\hline BGR-39 & H0209P08 & -86.3037 & 10.2965 & 1559 & 35.9 & 39.9 & 45.8 & $0.85^{\mathrm{f}}$ & 38.9 & HP1 \\
\hline BGR-39 & Н0209P09 & -86.3022 & 10.2963 & 1559 & 36.0 & 60.4 & 69.3 & $0.82^{\mathrm{e}}$ & 56.8 & HP1 \\
\hline BGR-39 & H0209P10 & -86.3005 & 10.2933 & 1614 & 35.9 & 51.9 & 50.8 & $0.79^{\mathrm{f}}$ & 40.1 & HP1 \\
\hline BGR-39 & H0209P11 & -86.2973 & 10.2902 & 1601 & 35.9 & 52.7 & 51.4 & $0.79^{\mathrm{e}}$ & 40.6 & HP1 \\
\hline BGR-39 & H0209P12 & -86.2973 & 10.2875 & 1610 & 35.7 & 47.2 & 47.2 & $0.78^{\mathrm{f}}$ & 36.8 & HP1 \\
\hline BGR-39 & H0209P13 & -86.2947 & 10.2937 & 1586 & 36.4 & 48.1 & 46.0 & $0.75^{\mathrm{e}}$ & 34.5 & HP1 \\
\hline BGR-39 & H0209P14 & -86.3153 & 10.3133 & 1660 & 36.3 & 52.9 & 52.0 & $0.80^{\mathrm{f}}$ & 41.6 & HP1 \\
\hline BGR-39 & H0210A01 & -86.3080 & 10.2985 & 1535 & 35.7 & 47.3 & 78.5 & $1.00^{\mathrm{e}}$ & 78.5 & MTL \\
\hline BGR-39 & H0211A01 & -86.3048 & 10.2998 & 1516 & 36.1 & 46.4 & 72.2 & $1.00^{\mathrm{e}}$ & 72.2 & MTL \\
\hline BGR-39 & H0212A01 & -86.3042 & 10.2968 & 1535 & 35.9 & 31.0 & 50.4 & $1.00^{\mathrm{e}}$ & 50.4 & MTL \\
\hline BGR-39 & H0213A01 & -86.3053 & 10.2945 & 1609 & 35.6 & 27.6 & 25.9 & $1.00^{\mathrm{e}}$ & 25.9 & MTL \\
\hline BGR-39 & H0214P01 & -86.4032 & 10.0873 & 3485 & 11.9 & 40.6 & 41.6 & $0.81^{\mathrm{f}}$ & 33.7 & HP1 \\
\hline BGR-39 & H0214P02 & -86.4180 & 10.0942 & 3545 & 11.3 & 51.0 & 52.1 & $0.78^{\mathrm{e}}$ & 40.6 & HP1 \\
\hline BGR-39 & H0214P03 & -86.3903 & 10.1005 & 3280 & 14.0 & 40.1 & 43.4 & $0.79^{\mathrm{f}}$ & 34.3 & HP1 \\
\hline BGR-39 & H0214P04 & -86.3838 & 10.1070 & 3258 & 15.0 & 45.3 & 43.3 & $0.78^{\mathrm{e}}$ & 33.8 & HP1 \\
\hline BGR-39 & H0214P05 & -86.3777 & 10.1133 & 3196 & 15.9 & 45.4 & 43.1 & $0.81^{\mathrm{f}}$ & 34.9 & HP1 \\
\hline BGR-39 & H0214P06 & -86.3710 & 10.1197 & 3083 & 17.0 & 46.3 & 43.8 & $0.78^{\mathrm{e}}$ & 34.2 & HP1 \\
\hline BGR-39 & H0214P07 & -86.3645 & 10.1262 & 2937 & 18.0 & 48.1 & 51.2 & $0.78^{\mathrm{f}}$ & 39.9 & HP1 \\
\hline BGR-39 & H0214P08 & -86.3582 & 10.1325 & 2864 & 19.0 & 55.0 & 47.8 & $0.78^{\mathrm{e}}$ & 37.3 & HP1 \\
\hline BGR-39 & Н0214P09 & -86.3517 & 10.1390 & 2694 & 20.0 & 46.9 & 49.9 & $0.78^{\mathrm{e}}$ & 38.9 & HP1 \\
\hline BGR-39 & H0214P10 & -86.3452 & 10.1455 & 2621 & 21.0 & 44.2 & 46.0 & $0.78^{f}$ & 35.9 & HP1 \\
\hline BGR-39 & H0215A01 & -86.3062 & 10.2977 & 1549 & - & - & - & $1.00^{\mathrm{e}}$ & - & MTL \\
\hline BGR-39 & H0216P01 & -86.3067 & 10.2982 & 1535 & 35.8 & 38.6 & 64.0 & $0.90^{\mathrm{f}}$ & 57.6 & HP1 \\
\hline BGR-39 & H0216P02 & -86.3062 & 10.2978 & 1549 & 35.8 & 39.0 & 59.7 & $0.90^{\mathrm{e}}$ & 53.7 & HP1 \\
\hline BGR-39 & H0216Р03 & -86.3062 & 10.2975 & 1549 & - & - & - & $0.90^{\mathrm{e}}$ & - & HP1 \\
\hline BGR-39 & H0218P01 & -86.5647 & 9.9288 & 4180 & -13.0 & 172.7 & 169.5 & $0.74^{\mathrm{f}}$ & 125.4 & HP1 \\
\hline BGR-39 & H0218P02 & -86.5580 & 9.9352 & 4178 & -12.0 & 169.6 & 165.3 & $0.75^{\mathrm{e}}$ & 124.0 & HP1 \\
\hline BGR-39 & Н0218Р03 & -86.5517 & 9.9412 & 4137 & -11.1 & 118.5 & 129.3 & $0.75^{\mathrm{e}}$ & 97.0 & HP1 \\
\hline BGR-44 & Н0219P01 & -86.0567 & 9.8030 & 2512 & 24.7 & 28.0 & 30.1 & $0.75^{\mathrm{e}}$ & 22.6 & HP1 \\
\hline BGR-44 & H0219P01 & -86.0567 & 9.8030 & 2512 & 24.7 & 28.0 & 30.1 & $0.75^{\mathrm{e}}$ & 22.6 & HP1 \\
\hline BGR-44 & H0219P02 & -86.0507 & 9.8097 & 2463 & 25.7 & 32.5 & 33.1 & $0.75^{\mathrm{e}}$ & 24.8 & HP1 \\
\hline BGR-44 & Н0219Р03 & -86.0447 & 9.8163 & 2407 & 26.7 & 32.0 & 31.8 & $0.51^{\mathrm{f}}$ & 16.2 & HP1 \\
\hline BGR-44 & Н0219P04 & -86.0388 & 9.8232 & 2371 & 27.6 & 34.9 & 32.5 & $0.75^{\mathrm{e}}$ & 24.4 & HP1 \\
\hline BGR-44 & H0220P01 & -86.0027 & 9.8640 & 1922 & 33.7 & 36.3 & 32.5 & $0.65^{\mathrm{f}}$ & 21.1 & HP1 \\
\hline BGR-44 & H0220P02 & -85.9968 & 9.8707 & 1806 & 34.7 & 24.3 & 22.3 & $0.75^{\mathrm{e}}$ & 16.7 & HP1 \\
\hline BGR-44 & H0220P03 & -85.9908 & 9.8777 & 1712 & 35.7 & 26.0 & 22.9 & $0.84^{\mathrm{f}}$ & 19.2 & HP1 \\
\hline BGR-44 & H0221A01 & -85.6802 & 9.8302 & 104 & - & - & - & $1.00^{\mathrm{e}}$ & - & MTL \\
\hline BGR-44 & H0221A02 & -85.6802 & 9.8303 & 104 & - & - & - & $1.00^{\mathrm{e}}$ & - & MTL \\
\hline BGR-44 & $\mathrm{H} 0222 \mathrm{P} 01$ & -86.2122 & 9.6265 & 4154 & -1.3 & 12.7 & 13.2 & $0.78^{\mathrm{e}}$ & 10.3 & HP1 \\
\hline BGR-44 & Н0222P02 & -86.2063 & 9.6330 & 4233 & -0.4 & 13.1 & 14.7 & $0.78^{\mathrm{e}}$ & 11.5 & HP1 \\
\hline BGR-44 & Н0222Р03 & -86.2005 & 9.6400 & 4345 & 0.7 & 14.0 & 13.4 & $0.82^{f}$ & 11.0 & HP1 \\
\hline BGR-44 & Н0222P04 & -86.1942 & 9.6467 & 4364 & 1.7 & 18.2 & 17.8 & $0.78^{\mathrm{e}}$ & 13.9 & HP1 \\
\hline BGR-44 & H0222P05 & -86.1885 & 9.6528 & 4379 & 2.6 & 19.6 & 15.6 & $0.77^{\mathrm{f}}$ & 12.0 & HP1 \\
\hline BGR-44 & Н0222Р07 & -86.1763 & 9.6673 & 4097 & 4.7 & 45.0 & 49.1 & $0.78^{\mathrm{e}}$ & 38.3 & HP1 \\
\hline BGR-44 & H0222P08 & -86.1703 & 9.6740 & 3981 & 5.7 & 11.9 & 12.5 & $1.44^{\mathrm{f}}$ & 18.0 & HP1 \\
\hline BGR-44 & Н0222P09 & -86.1647 & 9.6808 & 3888 & 6.7 & 19.0 & 19.1 & $0.78^{\mathrm{e}}$ & 14.9 & HP1 \\
\hline BGR-44 & $\mathrm{H} 0222 \mathrm{P} 10$ & -86.1585 & 9.6873 & 3785 & 7.6 & 26.4 & 28.0 & $0.87^{\mathrm{f}}$ & 24.4 & HP1 \\
\hline SO81-8 & H0223P01 & -85.9978 & 9.3980 & 3797 & -3.9 & 184.0 & 180.8 & $0.77^{\mathrm{f}}$ & 139.2 & HP1 \\
\hline SO81-8 & H0223P02 & -85.9927 & 9.4043 & 3824 & -3.0 & 167.4 & 167.5 & $0.77^{\mathrm{e}}$ & 129.0 & HP1 \\
\hline SO81-8 & H0223P03 & -85.9865 & 9.4117 & 3859 & -1.9 & 177.5 & 176.4 & $0.77^{\mathrm{e}}$ & 135.8 & HP1 \\
\hline SO81-8 & H0243P01 & -85.7928 & 9.6340 & 1660 & 30.7 & 43.0 & 42.9 & $0.77^{\mathrm{f}}$ & 33.0 & HP1 \\
\hline SO81-8 & Н0243P02 & -85.7987 & 9.6323 & 1687 & 30.1 & 43.3 & 44.5 & $0.78^{\mathrm{e}}$ & 34.7 & HP1 \\
\hline SO81-8 & H0243P03 & -85.8045 & 9.6253 & 1778 & 29.1 & 40.6 & 39.5 & $0.79^{\mathrm{f}}$ & 31.2 & HP1 \\
\hline SO81-8 & Н0243P04 & -85.8107 & 9.6185 & 1837 & 28.1 & 37.8 & 36.8 & $0.80^{\mathrm{e}}$ & 29.4 & HP1 \\
\hline
\end{tabular}


Table 1. (continued)

\begin{tabular}{|c|c|c|c|c|c|c|c|c|c|c|}
\hline Profile & Penetration & $\begin{array}{l}\text { Longitude } \\
\text { (deg) }\end{array}$ & $\begin{array}{l}\text { Latitude } \\
\text { (deg) }\end{array}$ & $\begin{array}{l}\text { Water } \\
\text { Depth } \\
(\mathrm{m})\end{array}$ & $\begin{array}{c}\text { Distance }^{\mathrm{a}} \\
(\mathrm{km})\end{array}$ & $\begin{array}{l}\text { Thermal } \\
\text { Gradient } \\
\left({ }^{\circ} \mathrm{C} \mathrm{km}^{-1}\right)\end{array}$ & $\begin{array}{l}\text { Corrected } \\
\text { Thermal } \\
\text { Gradient }^{\mathrm{b}} \\
\left({ }^{\circ} \mathrm{C} \mathrm{km}^{-1}\right)\end{array}$ & $\begin{array}{c}\text { Thermal } \\
\text { Conductivity } \\
\left(\mathrm{W} \mathrm{m}^{-1} \mathrm{~K}^{-1}\right)\end{array}$ & $\begin{array}{c}\text { Heat } \\
\text { Flow }^{\mathrm{c}} \\
\left(\mathrm{mW} \mathrm{m}^{-2}\right)\end{array}$ & Instrument $^{\mathrm{d}}$ \\
\hline SO81-8 & Н0243P05 & -85.8165 & 9.6280 & 1819 & 28.5 & 41.1 & 42.0 & $0.80^{\mathrm{f}}$ & 33.6 & HP1 \\
\hline SO81-8 & H0243P06 & -85.8222 & 9.6047 & 1928 & 26.1 & 28.8 & 31.1 & $0.80^{\mathrm{e}}$ & 24.9 & HP1 \\
\hline SO81-13 & H0226A01 & -84.9668 & 8.9268 & 3557 & -0.6 & 216.0 & 196.8 & $1.00^{\mathrm{e}}$ & 196.8 & MTL \\
\hline SO81-13 & H0228P01 & -84.8107 & 9.1733 & 785 & 31.0 & 43.3 & 44.3 & $0.89^{\mathrm{f}}$ & 39.4 & HP1 \\
\hline SO81-13 & H0228P02 & -84.8078 & 9.1735 & 773 & 31.2 & 51.6 & 52.5 & $0.89^{\mathrm{e}}$ & 46.7 & HP1 \\
\hline SO81-13 & H0228P03 & -84.8050 & 9.1737 & 761 & 31.5 & 65.3 & 68.5 & $0.89^{\mathrm{f}}$ & 61.0 & HP1 \\
\hline SO81-13 & H0228P04 & -84.8043 & 9.1735 & 761 & 31.5 & 48.3 & 50.7 & $0.89^{\mathrm{e}}$ & 45.1 & HP1 \\
\hline SO81-13 & H0228P05 & -84.8035 & 9.1738 & 753 & 31.6 & 20.7 & 21.7 & $0.90^{\mathrm{f}}$ & 19.5 & HP1 \\
\hline SO81-13 & H0228P06 & -84.8020 & 9.1738 & 743 & 31.7 & 52.5 & 57.1 & $0.89^{\mathrm{e}}$ & 50.8 & HP 1 \\
\hline SO81-13 & H0228P07 & -84.7987 & 9.1742 & 740 & 32.0 & 46.7 & 50.8 & $0.90^{\mathrm{f}}$ & 45.7 & HP1 \\
\hline SO81-13 & H0229P01 & -84.8242 & 9.1535 & 840 & 28.4 & 49.7 & 53.7 & $0.90^{\mathrm{f}}$ & 48.3 & HP1 \\
\hline SO81-13 & Н0229P02 & -84.8227 & 9.1525 & 827 & 28.4 & 49.7 & 55.6 & $0.89^{\mathrm{e}}$ & 49.5 & HP1 \\
\hline SO81-13 & H0229P04 & -84.8197 & 9.1505 & 848 & 28.5 & 54.0 & 53.5 & $0.86^{\mathrm{f}}$ & 46.0 & HP1 \\
\hline SO81-13 & H0229P05 & -84.8183 & 9.1497 & 888 & 28.5 & 45.6 & 38.9 & $0.89^{\mathrm{e}}$ & 34.6 & HP1 \\
\hline SO81-13 & H0229P06 & -84.8170 & 9.1485 & 860 & 28.5 & 49.5 & 49.7 & $0.91^{\mathrm{f}}$ & 45.2 & HP1 \\
\hline SO81-13 & H0229P07 & -84.8153 & 9.1475 & 890 & 28.6 & 40.6 & 36.3 & $0.89^{\mathrm{e}}$ & 32.3 & HP1 \\
\hline SO81-13 & H0229P08 & -84.8123 & 9.1458 & 889 & 28.7 & 50.1 & 49.8 & $0.89^{\mathrm{e}}$ & 44.3 & HP1 \\
\hline SO81-13 & H0229P09 & -84.8097 & 9.1438 & 872 & 28.7 & 34.1 & 34.1 & $0.91^{\mathrm{f}}$ & 31.0 & HP1 \\
\hline SO81-13 & H0229P10 & -84.8067 & 9.1422 & 863 & 28.8 & 34.7 & 34.9 & $0.89^{\mathrm{e}}$ & 31.1 & HP1 \\
\hline SO81-13 & H0241P01 & -84.8480 & 9.1140 & 2005 & 23.4 & 223.9 & 172.0 & $0.89^{\mathrm{e}}$ & 153.1 & HP1 \\
\hline SO81-13 & H0241P02 & -84.8417 & 9.1237 & 1712 & 24.7 & 201.2 & 156.7 & $0.89^{\mathrm{e}}$ & 139.5 & HP1 \\
\hline SO81-13 & H0241P03 & -84.8313 & 9.1272 & 1334 & 25.7 & 54.1 & 68.7 & $0.90^{\mathrm{f}}$ & 61.8 & HP1 \\
\hline SO81-13 & H0241P04 & -84.8368 & 9.1315 & 1390 & 25.7 & 174.9 & 143.0 & $0.89^{\mathrm{e}}$ & 127.3 & HP1 \\
\hline SO81-13 & H0241P05 & -84.8313 & 9.1403 & 948 & 26.8 & 40.8 & 49.6 & $0.92^{\mathrm{f}}$ & 45.6 & HP1 \\
\hline SO81-2 & H0227A01 & -84.8535 & 8.8283 & 3454 & -5.7 & 530.9 & 416.3 & $1.00^{\mathrm{e}}$ & 416.3 & MTL \\
\hline SO81-2 & $\mathrm{H} 0224 \mathrm{P} 01$ & -84.8508 & 8.8305 & 3461 & -5.3 & 516.7 & 444.7 & $0.81^{\mathrm{f}}$ & 360.2 & HP1 \\
\hline SO81-2 & H0224P02 & -84.8557 & 8.8235 & 3296 & -6.2 & 339.9 & 398.8 & $0.80^{\mathrm{e}}$ & 319.0 & HP1 \\
\hline SO81-2 & H0224P03 & -84.8600 & 8.8155 & 3272 & -7.2 & 395.1 & 414.3 & $0.80^{\mathrm{f}}$ & 331.4 & HP1 \\
\hline SO81-2 & H0224P04 & -84.8640 & 8.8077 & 3261 & -8.2 & 393.1 & 409.3 & $0.80^{\mathrm{e}}$ & 327.4 & HP1 \\
\hline SO81-2 & H0224P05 & -84.8685 & 8.7997 & 3263 & -9.2 & 316.8 & 291.4 & $0.78^{\mathrm{f}}$ & 227.3 & HP1 \\
\hline SO81-2 & $\mathrm{H} 0225 \mathrm{P} 01$ & -84.7957 & 8.9342 & 2882 & 7.7 & 77.0 & 66.1 & $0.82^{\mathrm{f}}$ & 54.2 & HP1 \\
\hline SO81-2 & H0225P02 & -84.7915 & 8.9422 & 2784 & 8.7 & 46.7 & 47.8 & $0.82^{\mathrm{e}}$ & 39.2 & HP1 \\
\hline SO81-2 & H0225P03 & -84.7872 & 8.9500 & 2758 & 9.7 & 56.3 & 52.0 & $0.80^{\mathrm{f}}$ & 41.6 & HP1 \\
\hline SO81-2 & H0225P05 & -84.7703 & 8.9823 & 2313 & 13.8 & 51.3 & 48.0 & $0.82^{\mathrm{f}}$ & 39.4 & HP1 \\
\hline SO81-2 & H0225P06 & -84.7662 & 8.9902 & 2151 & 14.7 & 62.6 & 66.2 & $0.82^{\mathrm{e}}$ & 54.3 & HP1 \\
\hline SO81-2 & H0225P07 & -84.7618 & 8.9982 & 2055 & 15.7 & 63.8 & 65.7 & $0.79^{\mathrm{f}}$ & 51.9 & HP1 \\
\hline SO81-2 & H0225P08 & -84.7573 & 9.0062 & 1931 & 16.8 & 70.6 & 69.8 & $0.82^{\mathrm{e}}$ & 57.2 & HP1 \\
\hline SO81-2 & H0225P09 & -84.7530 & 9.0142 & 1822 & 17.8 & 49.5 & 52.7 & $0.82^{\mathrm{f}}$ & 43.2 & HP1 \\
\hline SO81-2 & H0230P01 & -84.7015 & 9.1088 & 1232 & 29.7 & 35.8 & 37.0 & $0.89^{\mathrm{e}}$ & 32.9 & HP2 \\
\hline SO81-2 & $\mathrm{H} 0230 \mathrm{P} 02$ & -84.6972 & 9.1167 & 1188 & 30.7 & 36.5 & 36.6 & $0.89^{\mathrm{e}}$ & 32.6 & HP2 \\
\hline SO81-2 & $\mathrm{H} 0230 \mathrm{P} 03$ & -84.6928 & 9.1245 & 1149 & 31.7 & 34.3 & 34.5 & $0.89^{\mathrm{e}}$ & 30.7 & HP2 \\
\hline SO81-2 & H0230P04 & -84.6885 & 9.1325 & 1108 & 32.7 & 34.6 & 34.6 & $0.89^{\mathrm{e}}$ & 30.8 & HP2 \\
\hline SO81-2 & H0230P05 & -84.6840 & 9.1405 & 1082 & 33.7 & 33.0 & 31.6 & $0.89^{\mathrm{e}}$ & 28.1 & HP2 \\
\hline SO81-2 & H0230P06 & -84.6800 & 9.1483 & 1024 & 34.7 & 27.4 & 27.6 & $0.89^{\mathrm{e}}$ & 24.6 & HP2 \\
\hline SO81-2 & H0230Р07 & -84.6753 & 9.1570 & 984 & 35.8 & 29.2 & 28.4 & $0.89^{\mathrm{e}}$ & 25.3 & HP2 \\
\hline SO81-2 & H0230P08 & -84.6712 & 9.1645 & 897 & 36.8 & 32.0 & 35.2 & $0.89^{\mathrm{e}}$ & 31.3 & HP2 \\
\hline SO81-2 & H0230P09 & -84.6673 & 9.1727 & 857 & 37.8 & 23.8 & 21.2 & $0.89^{\mathrm{e}}$ & 18.9 & HP2 \\
\hline SO81-2 & H0231P01 & -84.8470 & 8.8392 & 3426 & -4.3 & 392.2 & 388.9 & $0.82^{\mathrm{e}}$ & 318.9 & HP1 \\
\hline SO81-2 & H0231P02 & -84.8427 & 8.8472 & 3445 & -3.2 & 403.0 & 421.0 & $0.83^{\mathrm{f}}$ & 349.4 & HP1 \\
\hline SO81-2 & H0231P03 & -84.8382 & 8.8550 & 3496 & -2.2 & 442.8 & 448.0 & $0.82^{\mathrm{f}}$ & 367.4 & HP1 \\
\hline SO81-2 & H0231P04 & -84.8345 & 8.8630 & 3521 & -1.3 & 331.5 & 353.0 & $0.82^{\mathrm{e}}$ & 289.5 & HP1 \\
\hline SO81-2 & H0231P05 & -84.8303 & 8.8708 & 3584 & -0.3 & 418.8 & 342.9 & $0.82^{\mathrm{e}}$ & 281.2 & HP1 \\
\hline SO81-2 & H0231P06 & -84.8260 & 8.8790 & 3433 & 0.7 & 229.6 & 228.8 & $1.03^{\mathrm{f}}$ & 235.7 & HP1 \\
\hline SO81-2 & Н0231P07 & -84.8215 & 8.8870 & 3398 & 1.8 & 115.9 & 108.5 & $0.88^{\mathrm{f}}$ & 95.5 & HP1 \\
\hline SO81-2 & H0231P08 & -84.8172 & 8.8950 & 3309 & 2.8 & 124.4 & 132.9 & $0.82^{\mathrm{e}}$ & 109.0 & HP1 \\
\hline SO81-2 & H0231P09 & -84.8132 & 8.8862 & 3406 & 2.1 & 130.7 & 115.5 & $0.82^{\mathrm{e}}$ & 94.7 & HP1 \\
\hline SO81-3 & H0233A01 & -84.6208 & 9.0335 & 1421 & 21.0 & 67.1 & 73.7 & $0.84^{\mathrm{e}}$ & 61.9 & MTL \\
\hline SO81-3 & H0234A01 & -84.6215 & 9.0340 & 1421 & 21.0 & 61.2 & 67.1 & $0.84^{\mathrm{e}}$ & 56.4 & MTL \\
\hline BGR17 & H0235P01 & -84.3072 & 8.9188 & 1074 & 33.5 & 61.4 & 58.3 & $0.90^{\mathrm{f}}$ & 52.5 & HP1 \\
\hline
\end{tabular}


Table 1. (continued)

\begin{tabular}{|c|c|c|c|c|c|c|c|c|c|c|}
\hline Profile & Penetration & $\begin{array}{l}\text { Longitude } \\
\text { (deg) }\end{array}$ & $\begin{array}{l}\text { Latitude } \\
\text { (deg) }\end{array}$ & $\begin{array}{l}\text { Water } \\
\text { Depth } \\
(\mathrm{m})\end{array}$ & $\begin{array}{c}\text { Distance }^{\mathrm{a}} \\
(\mathrm{km})\end{array}$ & $\begin{array}{l}\text { Thermal } \\
\text { Gradient } \\
\left({ }^{\circ} \mathrm{C} \mathrm{km}^{-1}\right)\end{array}$ & $\begin{array}{l}\text { Corrected } \\
\text { Thermal } \\
\text { Gradient }^{\mathrm{b}} \\
\left({ }^{\circ} \mathrm{C} \mathrm{km}^{-1}\right)\end{array}$ & $\begin{array}{c}\text { Thermal } \\
\text { Conductivity } \\
\left(\mathrm{W} \mathrm{m}^{-1} \mathrm{~K}^{-1}\right)\end{array}$ & 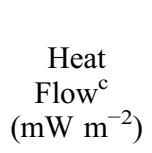 & Instrument $^{\mathrm{d}}$ \\
\hline BGR17 & H0235P02 & -84.3075 & 8.9207 & 1064 & 33.6 & 69.1 & 65.2 & $0.88^{\mathrm{e}}$ & 57.4 & HP1 \\
\hline BGR17 & Н0235Р03 & -84.3075 & 8.9223 & 1047 & 33.8 & 47.7 & 47.3 & $0.88^{\mathrm{e}}$ & 41.6 & HP1 \\
\hline BGR17 & H0235P04 & -84.3075 & 8.9240 & 1047 & 33.9 & 55.1 & 54.7 & $0.88^{\mathrm{e}}$ & 48.1 & HP1 \\
\hline BGR17 & H0235P05 & -84.3075 & 8.9257 & 1033 & 34.1 & 55.7 & 57.5 & $0.87^{\mathrm{f}}$ & 50.0 & HP1 \\
\hline BGR17 & Н0235Р06 & -84.3057 & 8.9192 & 1064 & 33.6 & 63.2 & 60.0 & $0.88^{\mathrm{e}}$ & 52.8 & HP1 \\
\hline BGR17 & Н0235P07 & -84.3057 & 8.9207 & 1055 & 33.8 & 68.0 & 64.5 & $0.88^{\mathrm{e}}$ & 56.8 & HP1 \\
\hline BGR17 & H0235P08 & -84.3057 & 8.9223 & 1035 & 33.9 & 71.1 & 75.5 & $0.88^{\mathrm{e}}$ & 66.4 & HP1 \\
\hline BGR17 & H0235P09 & -84.3057 & 8.9240 & 1035 & 34.1 & 67.2 & 71.2 & $0.89^{f}$ & 63.4 & HP1 \\
\hline BGR17 & $\mathrm{H} 0235 \mathrm{P} 10$ & -84.3057 & 8.9257 & 1033 & 34.2 & 61.0 & 60.3 & $0.88^{\mathrm{e}}$ & 53.1 & HP1 \\
\hline BGR17 & H0235P11 & -84.3042 & 8.9190 & 1064 & 33.7 & 29.2 & 27.7 & $0.88^{\mathrm{e}}$ & 24.4 & HP1 \\
\hline BGR17 & H0235P12 & -84.3042 & 8.9207 & 1055 & 33.8 & 73.6 & 69.9 & $0.88^{\mathrm{e}}$ & 61.5 & HP1 \\
\hline BGR17 & H0235P13 & -84.3040 & 8.9223 & 1024 & 34.0 & 71.0 & 81.3 & $0.88^{\mathrm{f}}$ & 71.5 & HP1 \\
\hline BGR17 & Н0235P14 & -84.3040 & 8.9242 & 1027 & 34.2 & 49.0 & 51.5 & $0.88^{\mathrm{e}}$ & 45.3 & HP1 \\
\hline BGR17 & H0235P15 & -84.3042 & 8.9258 & 1033 & 34.3 & 65.9 & 65.2 & $0.88^{\mathrm{e}}$ & 57.4 & HP1 \\
\hline BGR17 & H0235P16 & -84.3025 & 8.9257 & 1027 & 34.4 & 63.0 & 66.3 & $0.88^{\mathrm{e}}$ & 58.3 & HP1 \\
\hline BGR17 & $\mathrm{H} 0235 \mathrm{P} 17$ & -84.3023 & 8.9242 & 1027 & 34.3 & 64.4 & 67.7 & $0.87^{\mathrm{f}}$ & 58.9 & HP1 \\
\hline BGR17 & H0235P18 & -84.3023 & 8.9225 & 1024 & 34.1 & 70.2 & 80.3 & $0.88^{\mathrm{e}}$ & 70.7 & HP1 \\
\hline BGR17 & H0235P19 & -84.3023 & 8.9208 & 1039 & 34.0 & 71.0 & 73.8 & $0.82^{\mathrm{f}}$ & 60.5 & HP1 \\
\hline BGR17 & H0235P20 & -84.3023 & 8.9192 & 1047 & 33.8 & 71.9 & 73.9 & $0.88^{\mathrm{e}}$ & 65.0 & HP1 \\
\hline BGR17 & $\mathrm{H} 0235 \mathrm{P} 21$ & -84.3010 & 8.9192 & 1042 & 33.9 & 51.1 & 51.6 & $0.91^{\mathrm{f}}$ & 47.0 & HP1 \\
\hline BGR17 & H0235P22 & -84.3008 & 8.9207 & 1041 & 34.1 & 56.5 & 55.3 & $0.88^{\mathrm{e}}$ & 48.7 & HP1 \\
\hline BGR17 & H0235P23 & -84.3008 & 8.9223 & 1039 & 34.2 & 52.5 & 50.0 & $0.89^{f}$ & 44.5 & HP1 \\
\hline BGR17 & H0235P24 & -84.3007 & 8.9240 & 1039 & 34.4 & 50.6 & 48.2 & $0.88^{\mathrm{e}}$ & 42.4 & HP1 \\
\hline BGR17 & H0235P25 & -84.3007 & 8.9257 & 1035 & 34.5 & 51.4 & 49.3 & $0.87^{\mathrm{f}}$ & 42.9 & HP1 \\
\hline BGR17 & H0240A01 & -84.3043 & 8.9218 & 1055 & 33.9 & 8.8 & 8.3 & $0.90^{\mathrm{e}}$ & 7.5 & MTL \\
\hline BGR17 & H0240A02 & -84.3043 & 8.9218 & 1074 & - & - & - & $0.80^{\mathrm{e}}$ & - & MTL \\
\hline SO81-5 & Н0236Р01 & -84.3203 & 8.6513 & 2235 & 7.3 & 154.4 & 153.7 & $0.82^{f}$ & 126.0 & HP1 \\
\hline SO81-5 & H0236P02 & -84.3157 & 8.6588 & 2128 & 8.2 & 138.4 & 130.4 & $0.85^{\mathrm{e}}$ & 110.8 & HP1 \\
\hline SO81-5 & H0236P03 & -84.3105 & 8.6663 & 1969 & 9.3 & 106.7 & 109.1 & $0.85^{\mathrm{f}}$ & 92.7 & HP1 \\
\hline SO81-5 & Н0236Р04 & -84.3057 & 8.6740 & 1845 & 10.3 & 90.0 & 87.7 & $0.87^{\mathrm{e}}$ & 76.3 & HP1 \\
\hline SO81-5 & H0236P05 & -84.3008 & 8.6818 & 1749 & 11.3 & 77.4 & 71.1 & $0.85^{\mathrm{f}}$ & 60.4 & HP1 \\
\hline SO81-5 & H0236P06 & -84.2962 & 8.6895 & 1609 & 12.3 & 70.5 & 73.6 & $0.86^{\mathrm{e}}$ & 63.3 & HP1 \\
\hline SO81-5 & Н0236Р07 & -84.2913 & 8.6972 & 1545 & 13.3 & 83.0 & 83.3 & $0.86^{\mathrm{f}}$ & 71.6 & HP1 \\
\hline SO81-5 & Н0236Р08 & -84.2850 & 8.7047 & 1474 & 14.4 & 58.3 & 62.3 & $0.86^{\mathrm{e}}$ & 53.6 & HP1 \\
\hline SO81-5 & Н0236Р09 & -84.2818 & 8.7123 & 1409 & 15.3 & 59.8 & 58.1 & $0.86^{\mathrm{f}}$ & 50.0 & HP1 \\
\hline SO81-5 & H0236P10 & -84.2767 & 8.7203 & 1307 & 16.3 & 53.6 & 52.5 & $0.84^{\mathrm{e}}$ & 44.1 & HP1 \\
\hline SO81-5 & H0236P11 & -84.2720 & 8.7313 & 1137 & 17.6 & 58.8 & 59.8 & $0.84^{\mathrm{f}}$ & 50.2 & HP1 \\
\hline SO81-5 & H0237P01 & -84.3730 & 8.5673 & 2813 & -3.7 & 393.8 & 377.5 & $0.79^{f}$ & 298.2 & HP1 \\
\hline SO81-5 & H0237P02 & -84.3685 & 8.5750 & 2827 & -2.7 & 401.1 & 407.3 & $0.79^{\mathrm{e}}$ & 321.8 & HP1 \\
\hline SO81-5 & H0237P03 & -84.3635 & 8.5827 & 2849 & -1.7 & 282.8 & 283.7 & $0.78^{\mathrm{f}}$ & 221.3 & HP1 \\
\hline SO81-5 & H0237P04 & -84.3588 & 8.5903 & 2859 & -0.7 & 221.8 & 220.0 & $0.79^{\mathrm{e}}$ & 173.8 & HP1 \\
\hline SO81-5 & H0237P05 & -84.3540 & 8.5980 & 2893 & 0.3 & 200.8 & 183.5 & $0.80^{\mathrm{f}}$ & 146.8 & HP1 \\
\hline SO81-5 & H0237P06 & -84.3493 & 8.6055 & 2789 & 1.3 & 111.9 & 120.6 & $0.84^{\mathrm{e}}$ & 101.3 & HP1 \\
\hline SO81-5 & H0237P07 & -84.3442 & 8.6132 & 2725 & 2.3 & 122.7 & 118.8 & $0.86^{\mathrm{f}}$ & 102.2 & HP1 \\
\hline SO81-5 & H0237P08 & -84.3393 & 8.6210 & 2654 & 3.3 & 142.9 & 129.5 & $0.85^{\mathrm{e}}$ & 110.1 & HP1 \\
\hline SO81-5 & H0237P09 & -84.3347 & 8.6187 & 2640 & 3.3 & 138.9 & 129.7 & $0.88^{\mathrm{f}}$ & 114.1 & HP1 \\
\hline SO81-5 & $\mathrm{H} 0237 \mathrm{P} 10$ & -84.3300 & 8.6362 & 2428 & 5.3 & 194.9 & 190.1 & $0.87^{\mathrm{f}}$ & $\begin{array}{l}114.1 \\
165.4\end{array}$ & HP1 \\
\hline P1600 & Н0238Р01 & -84.1208 & 8.5005 & 1713 & 4.5 & 82.6 & 86.3 & $0.89^{\mathrm{f}}$ & 76.8 & HP1 \\
\hline P1600 & H0238P02 & -84.1247 & 8.4925 & 1866 & 3.5 & 84.4 & 82.2 & $0.87^{\mathrm{e}}$ & 71.5 & HP1 \\
\hline P1600 & H0238P03 & -84.1287 & 8.4845 & 2013 & 2.5 & 89.1 & 92.2 & $0.87^{\mathrm{f}}$ & 80.2 & HP1 \\
\hline P1600 & H0238P04 & -84.1327 & 8.4762 & 2190 & 1.5 & 186.4 & 169.3 & $0.83^{\mathrm{e}}$ & 140.5 & HP1 \\
\hline P1600 & H0238P05 & -84.1367 & 8.4680 & 2257 & 0.5 & 311.1 & 307.5 & $0.80^{\mathrm{f}}$ & 246.0 & HP1 \\
\hline P1600 & H0238P06 & -84.1407 & 8.4598 & 2229 & -0.5 & 249.6 & 263.3 & $0.80^{\mathrm{e}}$ & 210.6 & HP1 \\
\hline P1600 & Н0238P07 & -84.1448 & 8.4515 & 2291 & -1.6 & 162.0 & 136.2 & $0.81^{\mathrm{f}}$ & 110.3 & HP1 \\
\hline P1600 & H0238P08 & -84.1492 & 8.4433 & 2199 & -2.6 & 1072.0 & 962.8 & $0.85^{\mathrm{f}}$ & 818.4 & HP1 \\
\hline P1600 & Н0239P01 & -84.1210 & 8.5005 & & - & - & - & - & - & HP2 \\
\hline P1600 & H0239P02 & -84.1170 & 8.5085 & 1600 & 5.5 & 95.6 & 101.3 & $0.89^{\mathrm{e}}$ & 90.2 & HP2 \\
\hline P1600 & H0239P03 & -84.1130 & 8.5167 & 1499 & 6.5 & 82.4 & 84.5 & $0.89^{\mathrm{e}}$ & 75.2 & HP2 \\
\hline P1600 & Н0239P04 & -84.1090 & 8.5248 & 1421 & 7.5 & 116.0 & 108.8 & $0.89^{\mathrm{e}}$ & 96.8 & HP2 \\
\hline
\end{tabular}


Table 1. (continued)

\begin{tabular}{|c|c|c|c|c|c|c|c|c|c|c|}
\hline Profile & Penetration & $\begin{array}{l}\text { Longitude } \\
\text { (deg) }\end{array}$ & $\begin{array}{l}\text { Latitude } \\
\text { (deg) }\end{array}$ & $\begin{array}{l}\text { Water } \\
\text { Depth } \\
(\mathrm{m})\end{array}$ & $\begin{array}{l}\text { Distance }^{\mathrm{a}} \\
\quad(\mathrm{km})\end{array}$ & $\begin{array}{l}\text { Thermal } \\
\text { Gradient } \\
\left({ }^{\circ} \mathrm{C} \mathrm{km}^{-1}\right)\end{array}$ & $\begin{array}{l}\text { Corrected } \\
\text { Thermal } \\
\text { Gradient }^{\mathrm{b}} \\
\left({ }^{\circ} \mathrm{C} \mathrm{km}^{-1}\right)\end{array}$ & $\begin{array}{c}\text { Thermal } \\
\text { Conductivity } \\
\left(\mathrm{W} \mathrm{m}^{-1} \mathrm{~K}^{-1}\right)\end{array}$ & 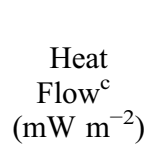 & Instrument $^{\mathrm{d}}$ \\
\hline P1600 & H0239P05 & -84.1052 & 8.5327 & 1289 & 8.5 & 76.5 & 80.4 & $0.89^{\mathrm{e}}$ & 71.6 & HP2 \\
\hline $\mathrm{P} 1600$ & H0239P06 & -84.1012 & 8.5408 & 1170 & 9.5 & 83.8 & 84.6 & $0.89^{\mathrm{e}}$ & 75.3 & HP2 \\
\hline $\mathrm{P} 1600$ & H0239P08 & -84.0928 & 8.5573 & 944 & 11.5 & 69.9 & 70.0 & $0.89^{\mathrm{e}}$ & 62.3 & HP2 \\
\hline P1600 & H0239P09 & -84.0890 & 8.5653 & 844 & 12.5 & 66.5 & 60.9 & $0.89^{\mathrm{e}}$ & 54.2 & HP2 \\
\hline P1600 & H0239P10 & -84.0850 & 8.5733 & 713 & 13.5 & 73.8 & 73.7 & $0.89^{\mathrm{e}}$ & 65.6 & HP2 \\
\hline
\end{tabular}

cruise M54/2 conducted in 2002. Heat flow measurements were collocated with seismic reflection profiles and swath bathymetry to aid interpretations (Figure 1). In general measurement spacing along transects is about $1 \mathrm{~km}$, but decreases to approximately $50 \mathrm{~m}$ around targets of interest. Heat flow measurements were made with a violin bow instrument design [Hyndman et al., 1979; Lister, 1979; Hartmann and Villinger, 2002] that allows measurements of both thermal gradient and thermal conductivity at multiple sites with a single transit to the seafloor. Two different violin bow instruments were used, a $3 \mathrm{~m}$ probe with 11 thermistors and a $6 \mathrm{~m}$ probe with 22 thermistors. The thermistors are equally spaced along the thermistor string. Additionally, a few heat determinations were made with autonomous temperature loggers attached to the side of gravity core barrels [Pfender and Villinger, 2002]. Heat flow determinations are processed based on the formulation described by Hartmann and Villinger [2002]. Each heat flow determination consists of inserting the probe into sediments under the force of gravity. Probe insertion generates a frictional heat pulse whose decay is well described by a cylindrical source. Equilibrium temperatures are computed by fitting the cylindrical decay curve to a $7 \mathrm{~min}$ period of data and then extrapolating to infinite time. In situ thermal conductivity was generally measured at every other site. These measurements are made following the initial $7 \mathrm{~min}$ period by generating a $20 \mathrm{~s}$ calibrated heat pulse [Lister, 1979] along a heater wire that extends over the length of the thermistor string. The temperature decay of the calibrated heat pulse provides a measure of sediment thermal conductivity at each thermistor. Data from each thermistor is monitored in real time through a coaxial cable between the instrument probe and the ship. Measured temperature gradients are linear indicating conductive heat transport at the resolution of the probe.

[19] Rapid sedimentation can transiently depress thermal gradients. Sedimentation rates estimated at DSDP Site 565 [von Huene et al., 1985] and ODP Site 1041 [Kimura et al., 1997] are similar. At Site 1041 [Kimura et al., 1997] sedimentation rates are $\sim 96 \mathrm{~m} / \mathrm{Myr}$ in the late Miocene, $62 \mathrm{~m} / \mathrm{Myr}$ in the early Pliocene, and $55 \mathrm{~m} / \mathrm{Myr}$ in the late Pliocene. A 1-D model of sedimentation and compaction [Wang and Davis, 1992] indicates that this depositional history depresses thermal gradients by less than a few percent; we do not correct for these small sedimentation effects in our heat flow determinations.

[20] Bathymetry has the potential to distort the thermal field by compressing isotherms under bathymetric lows and stretching them under bathymetric highs. Using a $\sim 225 \mathrm{~m}$ bathymetric grid [Ranero et al., 2008] and temperature-depth profiles through the water column from CTD casts we estimated a seafloor temperature distribution. This temperature distribution is diffused into the subsurface using Fourier series to calculate the thermal gradient due to the bathymetry following a procedure given by Blackwell et al. [1980]. With the exception of thermal gradients in the immediate vicinity of Culebra Mound [Grevemeyer et al., 2004], these three-dimensional perturbations generally produce only a small change in the thermal gradient (Table 1).

[21] In situ thermal conductivity values have a mean and standard deviation of $0.84 \mathrm{~W} \mathrm{~m}^{-1} \mathrm{~K}^{-1}$ 

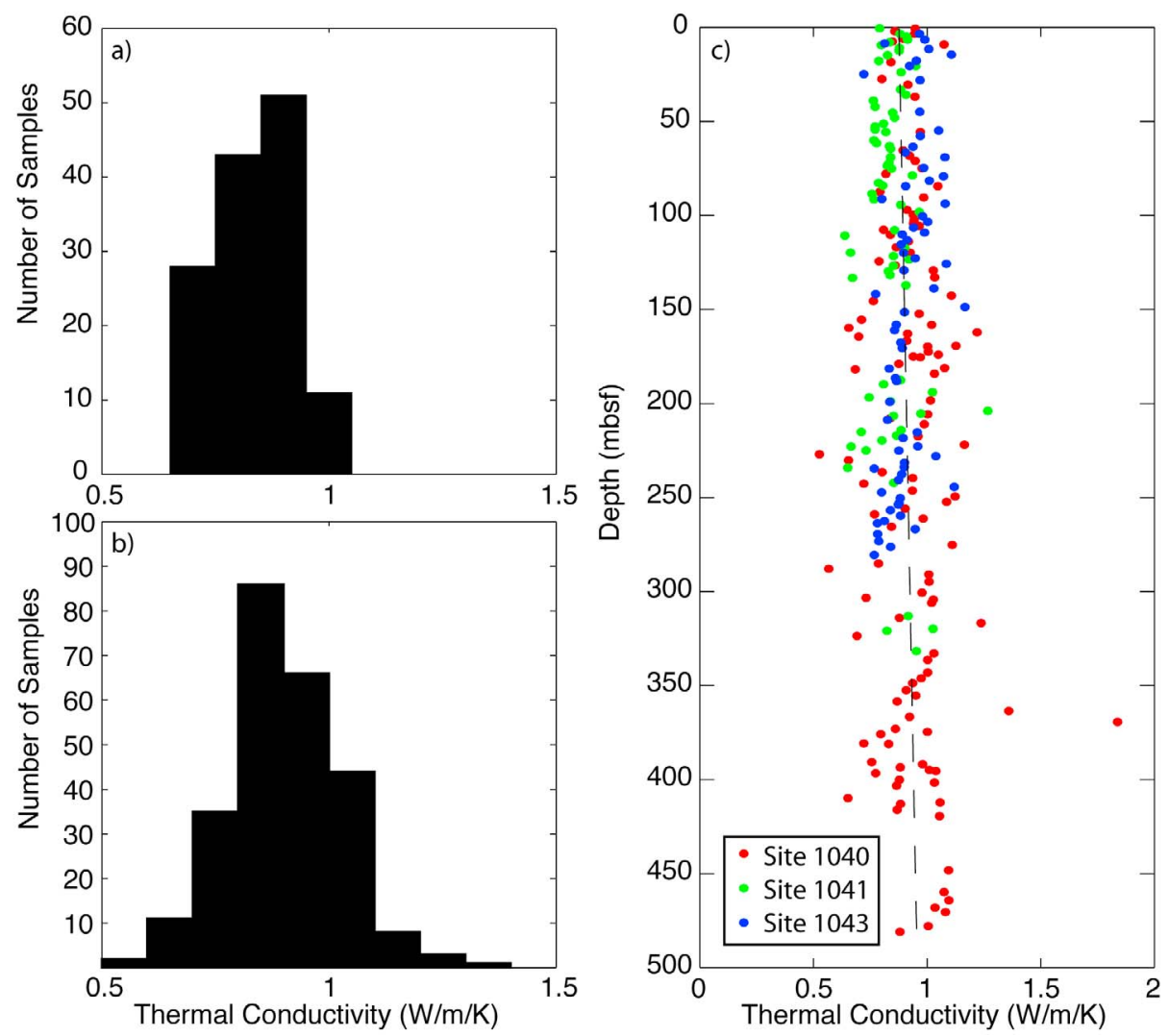

Figure 3. Thermal conductivity measurements. Histograms of thermal conductivity values from (a) shallow probes and (b) needle probe measurements ODP Leg 170 cores [Kimura et al., 1997] and (c) depth distribution of needle probe measurements.

and $0.08 \mathrm{~W} \mathrm{~m}^{-1} \mathrm{~K}^{-1}$, respectively (Figure 3a). No significant spatial variations in thermal conductivity are observed along strike. In situ values are only slightly lower than thermal conductivity values determined on ODP cores at sites 1040, 1041, and 1043 on the margin [Kimura et al., 1997]. These core values have a mean and standard deviation of 0.90 and $0.13 \mathrm{~W} \mathrm{~m}^{-1} \mathrm{~K}^{-1}$, respectively (Figure $3 \mathrm{~b}$ ). There is only a slight increase in thermal conductivity with depth (Figure 3c).

[22] Because observed thermal gradients are linear and thermal conductivity is approximately constant as a function of depth for each location, heat flow is computed as the product of the bathymetrically corrected thermal gradient and thermal conductivity (Table 1).

\section{BSR-Derived Heat Flow}

[23] The widespread presence of BSRs along the Costa Rica margin provides the opportunity to estimate geothermal gradients and when combined with estimates of thermal conductivity can extend the coverage of heat flow data. Gas hydrates are stable within the top few hundred meters of ocean floor sediments adjacent to continental margins, and the phase boundary between hydrate and free gas is marked by a bottom-simulating reflector (BSR) [e.g., Tucholke et al., 1977; Shipley et al., 1979; Grevemeyer et al., 2000; Pecher et al., 2001]. Within the zone of hydrate stability, pore space is occupied by solid hydrate and water, while below the zone of hydrate stability, pore space is occupied by free gas and water. The transition between hydrate and water to free gas and water produces an acoustic impedance contrast that often generates a well-developed high-amplitude, reversed polarity reflection that is relatively straightforward to identify. Because the base of the hydrate stability field is primarily sensitive to temperature, with only moderate sensitivity to pressure and composition, BSRs correspond to an isotherm and provide a way to map regional estimates of average geothermal gradients on continental margins [e.g., Hyndman et al., 1992; Brown and Bangs, 1995; Kaul et al., 2000; 
Grevemeyer and Villinger, 2001; Minshull et al., 2005]. BSR depths are converted to estimates of heat flow based on the approach developed by Yamano et al. [1982] and reviewed in detail by Grevemeyer and Villinger [2001]. Heat flow is calculated as,

$$
q=k \frac{\left(T_{b s r}-T_{\text {sea }}\right)}{\left(z_{b s r}-z_{\text {sea }}\right)},
$$

where $k$ is the thermal conductivity, $T_{b s r}$ is the temperature at the BSR, $T_{\text {sea }}$ is the temperature of the seafloor, $z_{b s r}$ is the depth of the BSR and $z_{\text {sea }}$ is the depth of the seafloor. This simple equation, however, is only valid for the case of a constant thermal conductivity between seafloor and BSR as indicated here (Figure 3).

[24] The geothermal gradient is based on the depth interval between the seafloor and the BSR and difference between the estimated bottom water temperature and the temperature at the BSR. Travel times between the seafloor and BSR were estimated by identifying the positive peak at seafloor and the negative peak at the BSR. The picking error is typically $<20 \mathrm{~ms}$, or about $5 \%-10 \%$. A simple linear velocity function was used to convert this two way travel time to depth based on depth migrated seismic lines (SO81-4, SO81-5, SO81-17a, and BGR99-7),

$$
\mathrm{v}=1550+0.309 \mathrm{z}
$$

where $v$ is the $\mathrm{P}$ wave velocity in $\mathrm{m} / \mathrm{s}$ and $z$ is the depth below seafloor in $\mathrm{m}$. Although this simplified model neglects second-order velocity variations such as the potential velocity increase above the BSR in regions of strong hydrate concentration, the calculated heat flow is not very sensitive to seismic velocity. An increase in BSR depth would also increase the temperature at the BSR such that the overall change in the thermal gradient is small [Grevemeyer and Villinger, 2001].

[25] Seafloor temperatures, $T_{\text {sea }}$, are estimated based on CTD casts from the NOAA World Ocean Data Atlas and the depth of the seafloor above each BSR pick. Seafloor temperature is assumed to be a function of depth only. Over the region of gas hydrates seafloor temperatures vary between $2^{\circ} \mathrm{C}$ and $15^{\circ} \mathrm{C}$ as a function of depth. Temporal variations in bottom water temperature are not observed. Heat probe determinations at water depths of $\sim 1000 \mathrm{~m}$ show little evidence of nonlinear gradients that might reflect temporal variations in bottom water temperatures. ODP sites 1253 and 1255 with water depths of 4312 and 4376, respectively, show annual changes less than $0.1^{\circ} \mathrm{C}$ [Davis and Villinger, 2006].

[26] The temperature at the BSR dept is calculated assuming pressure-temperature stability conditions for methane hydrate and that the primary composition is methane in seawater. We use the Dickens and Quinby-Hunt [1994] relationship,

$$
\frac{1}{T}=3.79 \cdot 10^{-3}-2.83 \cdot 10^{-4} \log (P)
$$

where $T$ is the temperature in $\mathrm{K}$ and $P$ is the pressure in $\mathrm{MPa}$. Pressure at the BSR is computed assuming hydrostatic conditions and a seawater density of $1030 \mathrm{~kg} / \mathrm{m}^{3}$.

[27] Grevemeyer and Villinger [2001] show that sediment thermal conductivity is not sensitive to the presence of gas hydrates. Based on numerical models for ODP Site 1040, Hensen and Wallmann [2005] estimate the concentration of hydrate at 1.65 volume percent of pore space. Grevemeyer and Villinger [2001] reviewed thermal conductivity, porosity and logging data from ODP sites drilled into and through gas hydrates. They find that although porosity decreases with depth, increasing the seismic velocity, thermal conductivity profiles between the seafloor and BSR can be fit using a mean value about as well as one that increases slightly with depth. Based on the lack of evidence for spatial variation in thermal conductivity along the margin and the relatively constant values of thermal conductivity determined from ODP Leg 170 , we assumed a constant thermal conductivity of $0.9 \mathrm{~W} \mathrm{~m}^{-1} \mathrm{~K}^{-1}$ (Figure 3). We estimate the total error (root mean square of all errors) may reach $10 \%-20 \%$ and a relative error of approximately $5 \%-10 \%$.

\section{Comparisons Between in Situ Heat Flow and BSR-Derived Estimates of Heat Flow}

[28] Heat flow observed with shallow probes and derived from BSRs are sensitive to different length scales. The 3 and $6 \mathrm{~m}$ probes used in this study have greater sensitivity to shallow thermal disturbances such erosion and/or sedimentation than BSR-derived values of heat flow. In contrast, BSRderived values of heat flow are more sensitive to deeper thermal processes within the margin and their use in calculating heat flow assumes that the BSR is in thermal equilibrium. Advective processes generating curvature in the thermal gradient, such 

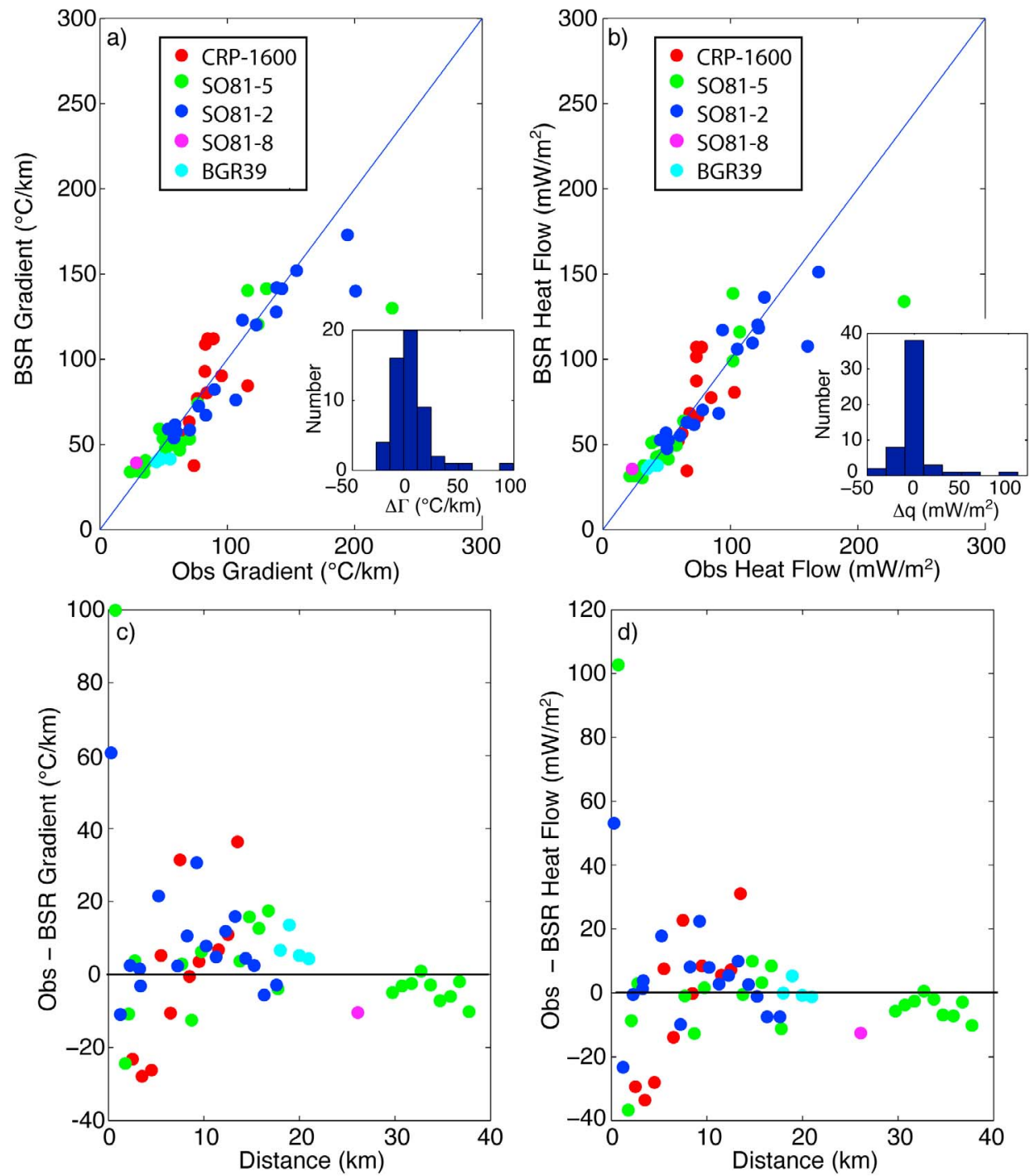

Figure 4. Correlations between probe and BSR-derived values of (a) thermal gradient and (b) heat flow. Correlations are color coded by seismic line. Insets show histograms of differences in thermal gradient and heat flow. (c and d) Thermal gradient and heat flow differences as a function of distance from the deformation front. The differences generally decrease with landward distance from the deformation front.

as block sliding or rotation, would lead to incorrect estimates of BSR-derived heat flow.

[29] Figure 4 shows comparisons between probe and BSR-derived values of thermal gradient and heat flow. These correlations are based on probe measurements within $2.5 \mathrm{~km}$ of the closest BSR determination. Correlations between the thermal gradient and heat flow both have $r^{2}$ values of approximately 0.80 (Figures $4 \mathrm{a}$ and $4 \mathrm{~b}$ ). A histogram of differences between probe and BSR heat flow values (Figure 4b, inset) show that differences are slightly skewed toward higher probe values of heat flow. This comparison contrasts with other studies [e.g., Ruppel, 1997; Kaul et al., 2000] where BSR-derived values of heat flow are larger than probe values. In general values of heat flow agree to about $10 \%$.

[30] Differences between probe and BSR values of heat flow are correlated with proximity to the deformation front (Figures $4 \mathrm{c}$ and $4 \mathrm{~d}$ ). Within about $5 \mathrm{~km}$ of the deformation front probe values of heat flow are slightly less than BSR values and between 5 and $15 \mathrm{~km}$ probe values of heat flow are generally somewhat higher than BSR-derived values. When points within $5 \mathrm{~km}$ of the deformation front are excluded, the standard deviation of 

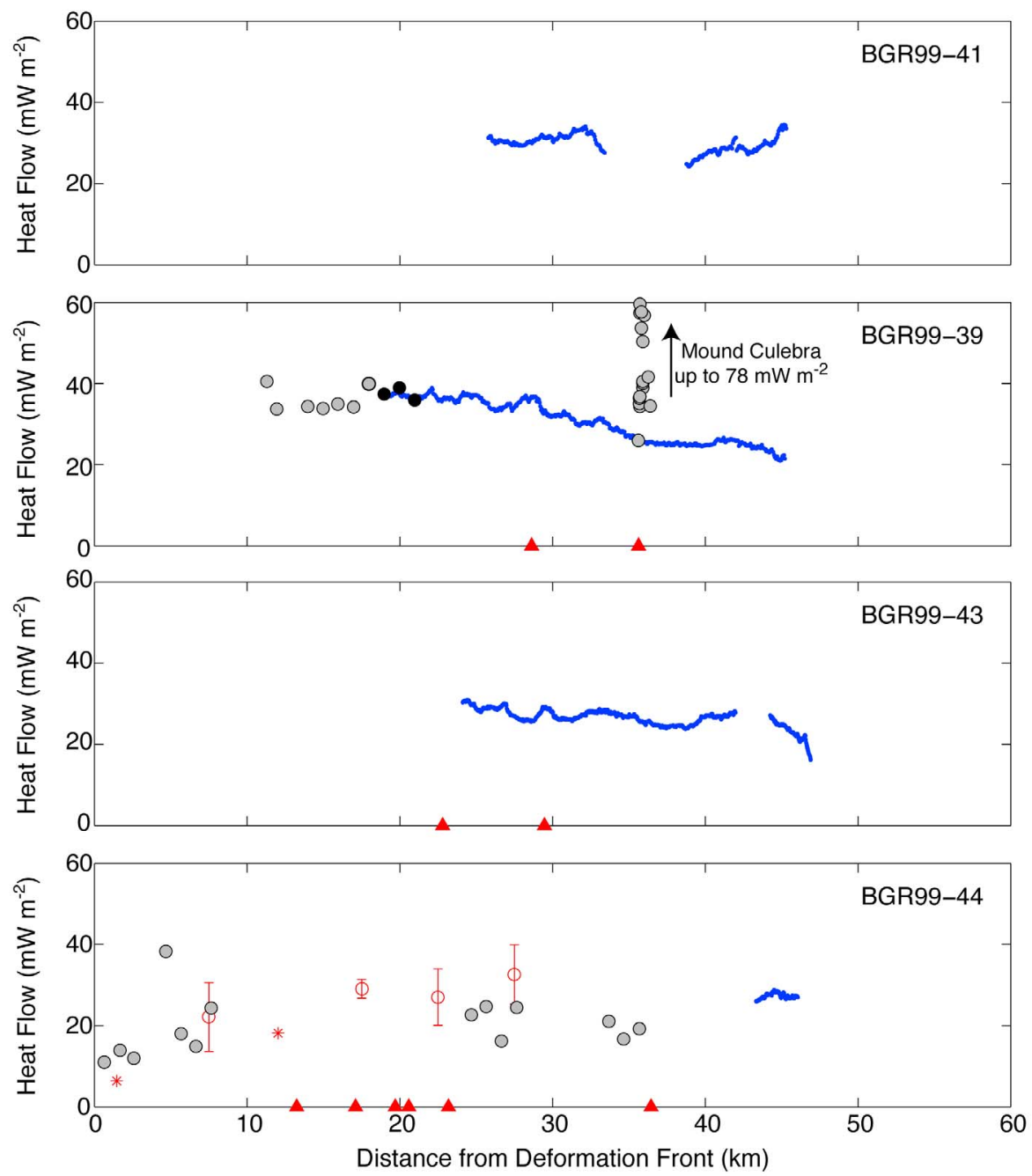

Figure 5. Heat flow profiles offshore the northern Nicoya Peninsula showing observed (black circles) and BSRderived (small blue circles) values of heat flow. Solid black circles denote heat flow values within $2.5 \mathrm{~km}$ of a BSR value. Heat flow values from Langseth and Silver [1996] averaged over $5 \mathrm{~km}$ windows are shown as circles with error bars representing the standard deviation. ODP values of heat flow are shown as stars [Kimura et al., 1997]. The positions of seeps along each profile are denoted as solid triangles at the base of each plot [Sahling et al., 2008].

the differences decreases from 28 to $10 \mathrm{~mW} / \mathrm{m}^{2}$. The major source of these differences is likely thermal disequilibrium caused by deformation.

\section{Discussion}

[31] The generally good correspondence between the probe and BSR-derived values of heat flow suggests that BSR-derived values of heat flow are well determined and can be used with confidence to extend thermal mapping of the margin. Most comparisons between BSR- and probe-derived values have been made at accretionary prisms such as Cascadia [Davis et al., 1990], Makran [Kaul et al., 2000], and Nankai [Yamano et al., 1982] and are generally consistent to about $15 \%-20 \%$ [Hyndman et al., 1992; Grevemeyer and Villinger, 2001].

[32] The good agreement between probe and BSRderived values of heat flow suggest that this margin may be thermally more stable than these accretionary margins. The thermal evolution of the erosional Central America margin differs from that of Nankai, Makran, and Cascadia. Here, the crystalline rocks now beneath the middle slope of the margin subsided 3-5 km in 1-2 Ma because of erosional thinning [Vannucchi et al., 2003]. In this manner the upper margin is transported into the lower margin temperature field. In contrast, at accretionary prisms, ocean basin sediment that has been drained of its pore water in the frontal prism 

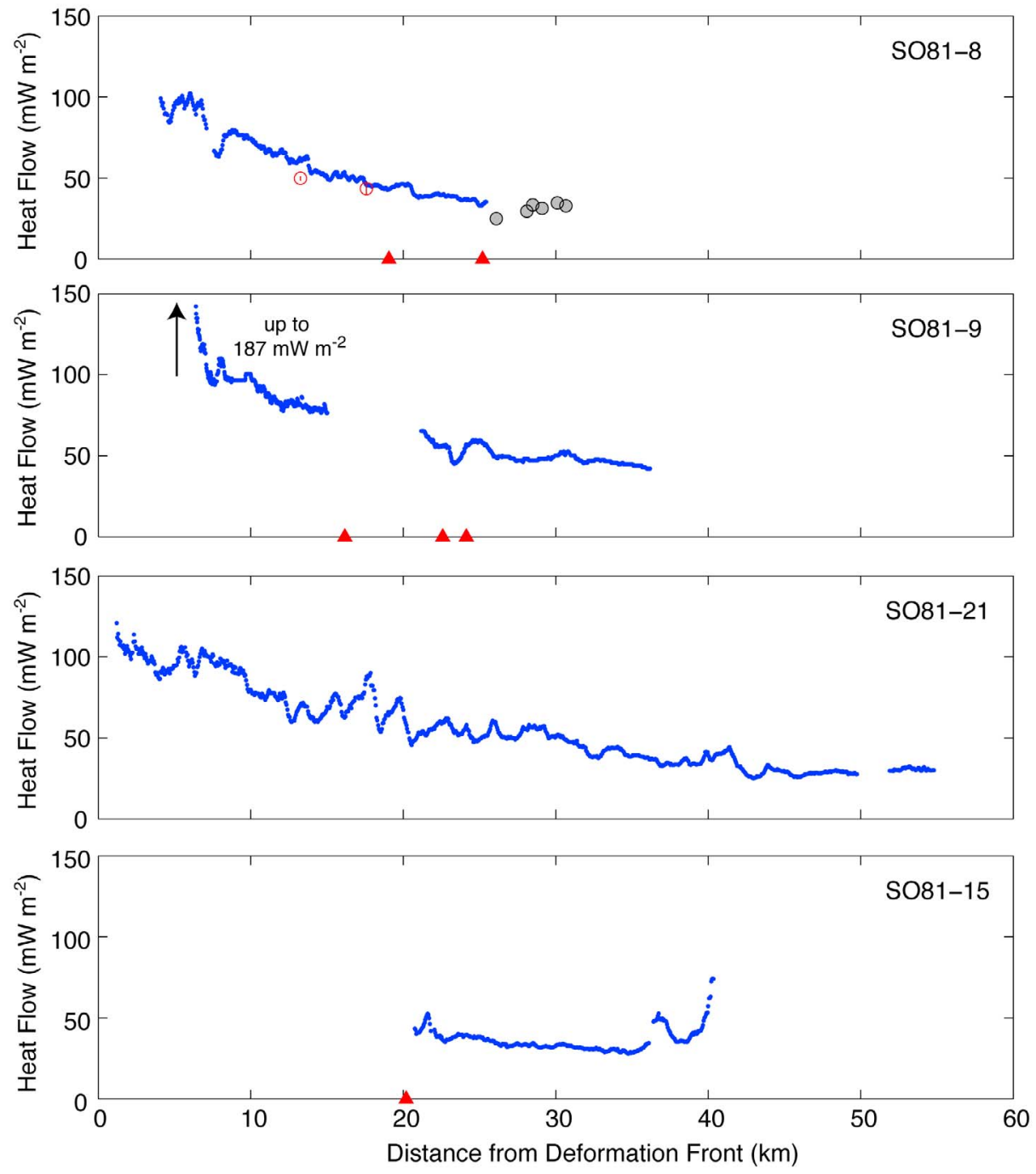

Figure 6. Heat flow profiles offshore the southern Nicoya Peninsula showing observed (solid circles) and BSRderived (small circles) values of heat flow. Heat flow values from Langseth and Silver [1996] averaged over $5 \mathrm{~km}$ windows are shown as circles with bars showing standard deviation. The positions of seeps along each profile are denoted as solid triangles at the base of each plot [Sahling et al., 2008].

becomes the middle and upper slope and is transported into the upper slope temperature field.

[33] Figures 5-8 show transects of both the in situ and BSR-derived values of heat flow along seismic lines. In general heat flow decreases with distance from the deformation front consistent with the subducting plate cooling the margin as it advects heat toward the Earth's interior.

[34] Heat flow transects along the northern portion of the Nicoya segment (Figure 5) are relatively low with maximum values less than $\sim 40 \mathrm{~mW} \mathrm{~m}^{-2}$. These values of heat flow are remarkably low and well below the $95-105 \mathrm{~mW} \mathrm{~m}^{-2}$ expected for 24 Ma crust. A notable exception, however, is the locally high heat flow associated with the seepage site of Culebra Mound [Grevemeyer et al., 2004] that lies a few kilometers north of seismic line BGR99-39. The absence of BSRs within approximately $20 \mathrm{~km}$ of the deformation front may indicate low heat flow that places the BSR depth within the oceanic crust, as suggested by deep temperature measurements made on ODP Leg 170 [Ruppel and Kinoshita, 2000], or within the ophiolitic rocks making up the margin. For example, the seaward extent of the BSR along seismic line BGR99-39 appears to be truncated where it intersects the basement rocks of the margin (Figure 2a). The magnitude of heat flow values and their pattern are consistent with exceptionally cold oceanic crust cooling the margin [Fisher et al., 2003; Hutnak et al., 2007]. 

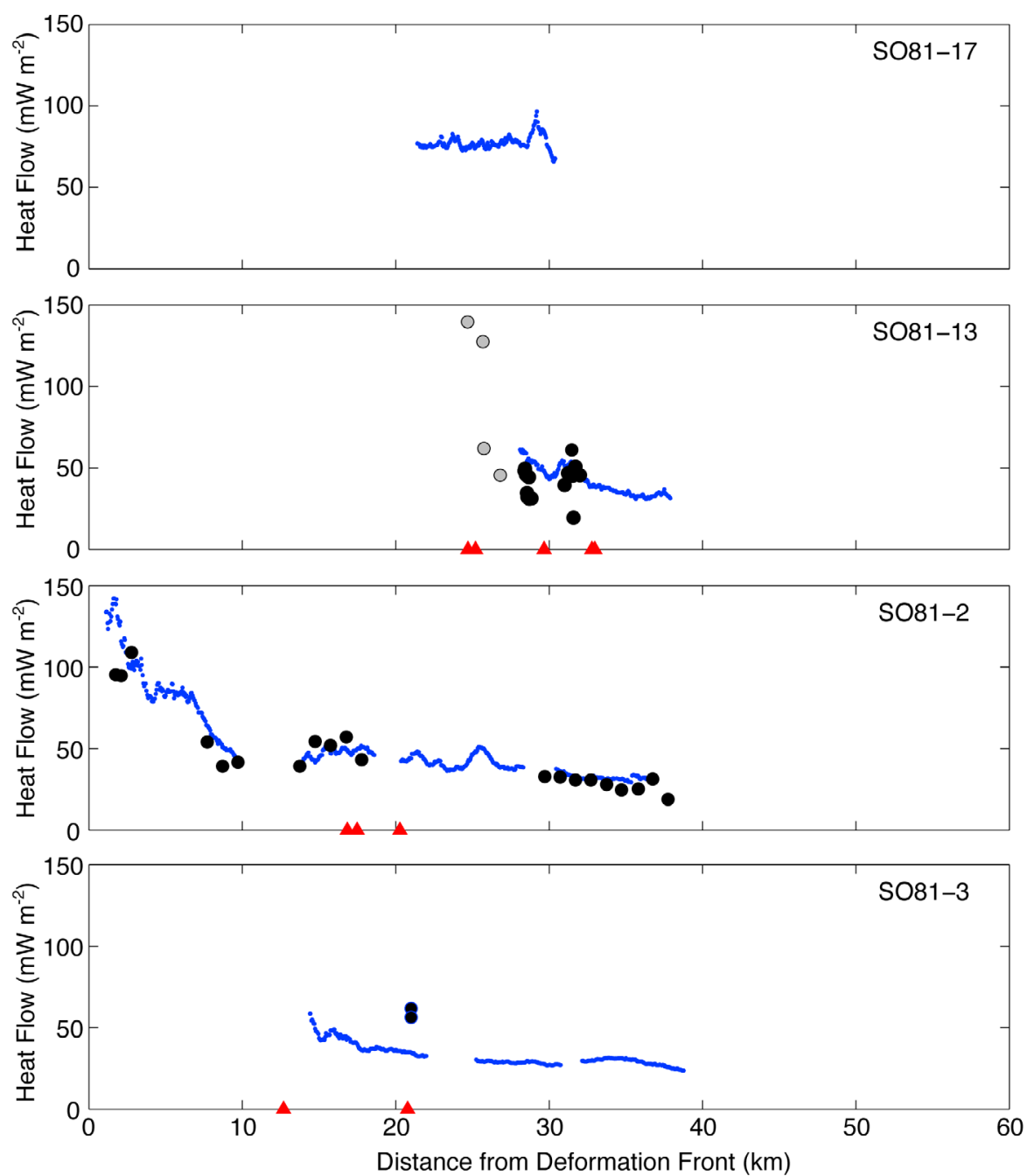

Figure 7. Heat flow profiles within the seamount segment showing observed (black circles) and BSR-derived (blue circles) values of heat flow. Solid black circles denote heat flow values within $2.5 \mathrm{~km}$ of a BSR value. The positions of seeps along each profile are denoted as solid triangles at the base of each plot [Sahling et al., 2008].

[35] Heat flow transects along the southern Nicoya Peninsula (Figure 6) are predominantly based on BSRs. Comparisons of BSR-derived estimates of heat flow and in situ values along seismic line SO81-8 are consistent with each other. Near the deformation front heat flow values are approximately $100 \mathrm{~mW} \mathrm{~m}^{-2}$ and decrease landward. BSRs along seismic line SO81-15 are truncated by a slump headwall (Figure 2c).

[36] Heat flow transects within the Seamount segment show large variability (Figure 7). Heat flow along profiles SO81-17 and SO81-13 are only available for a short distance because of the disrupted margin. In situ heat flow values along profile SO81-13 are highly variable over a short distance likely due to the advective influence of seeps that was a target of this heat flow station.
Profile SO81-2 has the largest data span across the margin. Near the deformation front heat flow values are in excess of $100 \mathrm{~mW} \mathrm{~m}{ }^{-2}$ and then decrease sharply to about $50 \mathrm{~mW} \mathrm{~m}{ }^{-2} 10 \mathrm{~km}$ landward of the deformation front. In situ values of heat flow agree well with BSR estimates of heat flow.

[37] Heat flow profiles corresponding to the Cocos Ridge segment of the margin (Figure 8) display some of the highest observed values of heat flow along the margin. Within $5 \mathrm{~km}$ of the deformation front, heat flow is quite high and locally exceeds values of $150 \mathrm{~mW} \mathrm{~m}^{-2}$. Along profile BGR99-17 heat flow values targeted Mound 11 [Schmidt et al., 2005; Sahling et al., 2008] and are quite variable. A pronounced heat flow high centered about $7 \mathrm{~km}$ landward of the deformation front is observed 

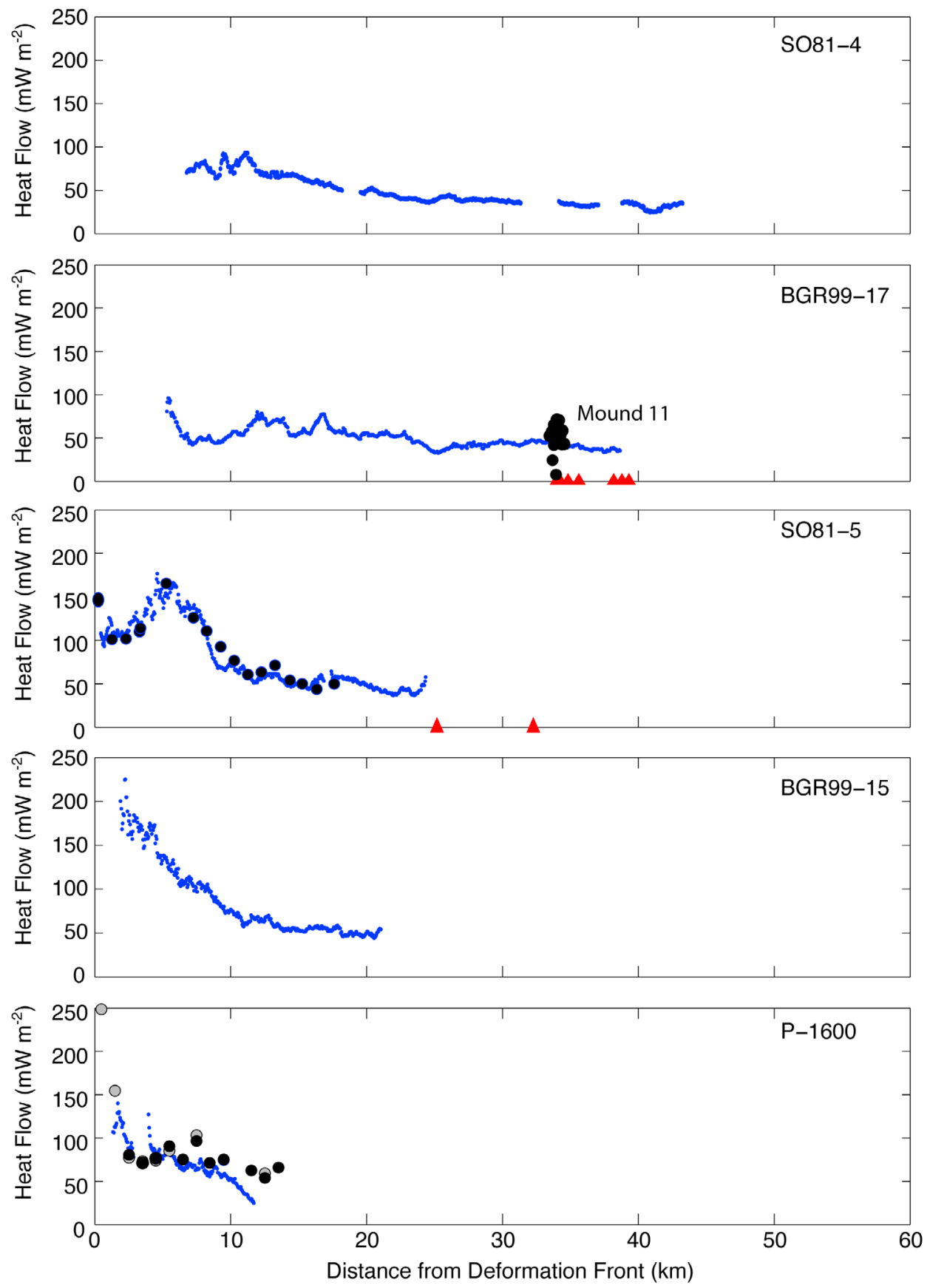

Figure 8. Heat flow profiles within the Cocos Ridge segment showing observed (black circles) and BSR-derived (blue circles) values of heat flow. Solid black circles denote heat flow values within $2.5 \mathrm{~km}$ of a BSR value. The positions of seeps along each profile are denoted as solid triangles at the base of each plot [Sahling et al., 2008].

along profile SO81-5. This feature correlates well with a seismically imaged horst in the downgoing oceanic plate and may reflect hydrothermal circulation within this feature.

[38] Hand drawn contours of heat flow data (Figure 9) show two thermally distinct regions. The margin offshore northern Nicoya Peninsula is rel- atively cool, especially in the area of seismic line BGR99-44 where heat flow increases landward of the deformation front before decreasing [Langseth and Silver, 1996]. This area is likely a continuation of the cool section of the Cocos plate mapped by Fisher et al. [2003]. To the south of the plate suture (Figure 1), heat flow increases sharply. This new data extends the transition between the warmer 


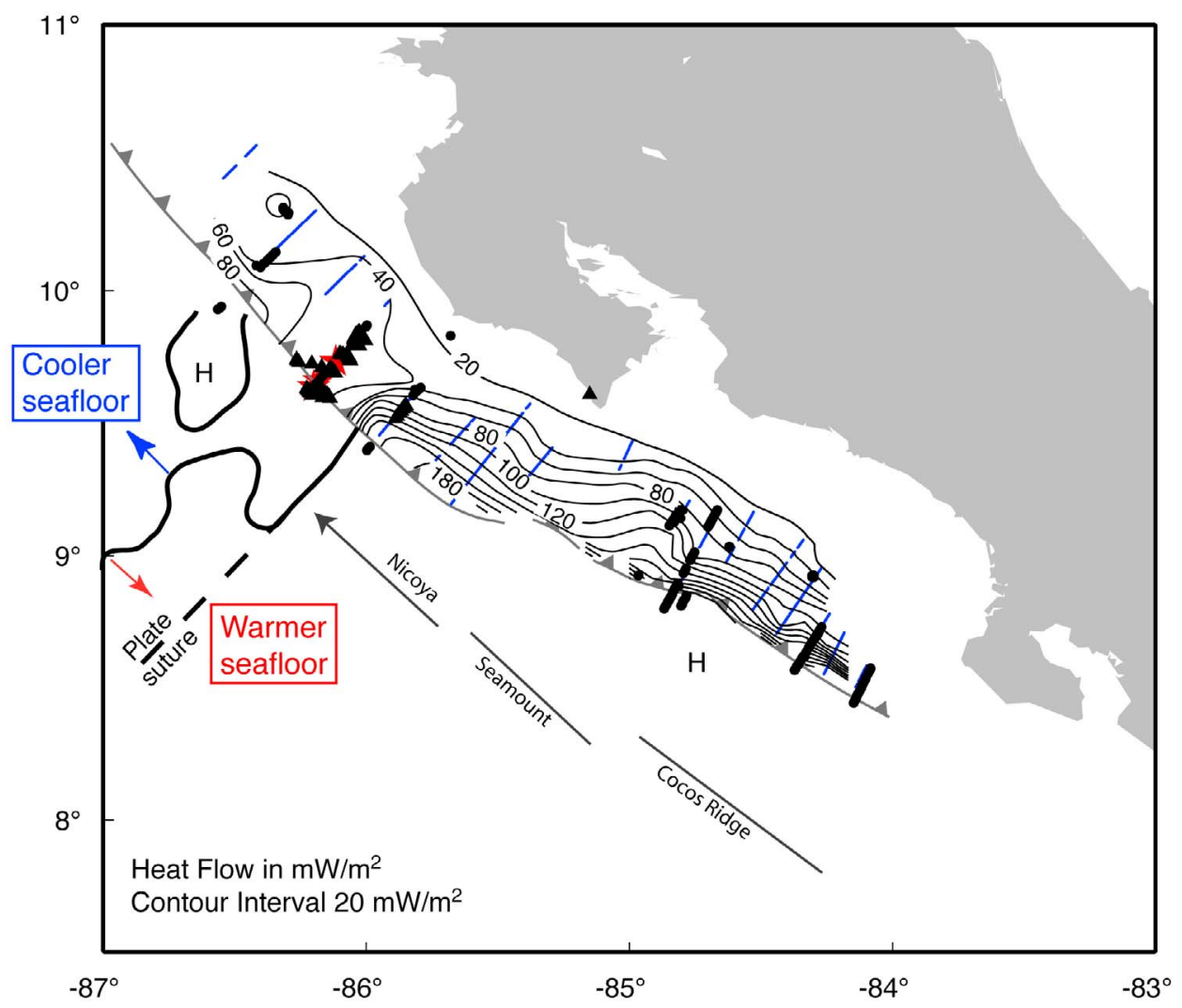

Figure 9. Heat flow map of the Costa Rica margin. Contours are in intervals of $20 \mathrm{~mW} \mathrm{~m}^{-2}$. Blue lines show location of BSR-derived heat flow, and solid circles show probe values of heat flow. Triangles show data of Langseth and Silver [1996], and stars show ODP values of heat flow [Ruppel and Kinoshita, 2000]. The boundary between normal and cold EPR crust is from Hutnak et al. [2008].

and cooler oceanic crust, mapped by Fisher et al. [2003] and Hutnak et al. [2007] seaward of the deformation front. Between seismic lines SO81-15 and SO81-13 the margin is disrupted by seamount subduction and there is a relatively large gap in thermal data. Here a large seamount has begun to tunnel into the margin and is marked by considerable slope failure and gravity sliding. An additional buried seamount is uplifting the margin forming Cabo Blanco with rapidly uplifted shorelines [Gardner et al., 2001]. Contours are interpolated through this area and are less precisely located than to the north and south. In the southern seamount segment and Cocos Ridge segment heat flow is anomalously high and decreases rapidly landward.

[39] Another feature of the data are high-wave number variations approximately 5 to $10 \mathrm{~km}$ wide (Figures 5-8). These variations are most pronounced where rough seafloor is being subducted south of the Nicoya segment (Figure 6). Although, steady state erosion and sedimentation are often used to explain heat flow fluctuations, the scale of the variability and its association with subduction basement relief leads us toward alternative explanations. Candidate processes that may cause the observed variations are heat refraction caused be variations in basement relief, advective fluid flow, and the effects of deformation.

[40] In general we do not observe a positive correlation between heat flow and basement relief that suggests to us that heat refraction is a dominant process leading to the observed variability. For example seismic line SO81-21 shows a prominent basement high at a distance of approximately $16 \mathrm{~km}$ from the deformation front (Figure 2b). Heat flow over this feature oscillates at wave numbers significantly less than its observed width of about $4 \mathrm{~km}$ (Figure 6). To assess the impact of heat refraction, we parameterize basement as a sinusoid with a wave number and amplitude of 4 and $1 \mathrm{~km}$, respectively, and include sediment cover with a minimum thickness of $0.5 \mathrm{~km}$. The basement thermal conductivity may be as high as $3 \mathrm{~W} \mathrm{~m}^{-1}$ $\mathrm{K}^{-1}$ and the overlying sediments may be about 
$0.9 \mathrm{~W} \mathrm{~m}^{-1} \mathrm{~K}^{-1}$, so that the ratio of thermal conductivity is about $3: 1$. The maximum heat flow variability generated by this model is about $15 \%$. This numerical model indicates that while heat refraction may contribute to the observed heat flow variability, it is in general not a large component.

[41] Fluid flow through the margin is a likely source of high wave number variability. Two major pathways of fluid flow associated with the Costa Rican margin are thought to exist. The first is along the decollement [Saffer et al., 2000; Silver et al., 2000] and the second is through normal faults that penetrate the margin [Hensen et al., 2004; Sahling et al., 2008; Ranero et al., 2008]. Near the deformation front, fluid flow is driven by compaction loading of the overriding margin. Saffer et al. [2000] estimated a fluid volume flux of $\sim 8 \mathrm{~m}^{3} / \mathrm{yr}$ per meter of trench length based on correlations of borehole density logs between reference ODP Sites 1039 and 1043, $1.6 \mathrm{~km}$ landward of the deformation front. Flow is thought to occur upward from the underthrust sediments to the base of the décollement zone and then through a thin zone of sediments just below the décollement [Screaton and Saffer, 2005]. The fast convergence rate coupled with the subduction of all of the incoming $\sim 400 \mathrm{~m}$ thick sediment section suggests that fluid flow may be more sustained than at other subduction zones [Screaton and Saffer, 2005].

[42] In addition to pore fluid expulsion, fluids are generated through the dehydration of hydrous minerals and phase transformations at depth. Geochemical measurements of pore waters show low values of chlorinity and salinity consistent with fluid production through dehydration reactions at depth [Chan and Kastner, 2000; Hensen and Wallmann, 2005]. These fluids may preferentially flow through normal faults that penetrate the margin. Observations of seafloor seepage mapped at 124 sites are summarized by Sahling et al. [2008]. They find that mapped seeps are concentrated within a band 17 to $40 \mathrm{~km}$ from the deformation front. Along the Nicoya segment and to the north, the majority of seeps are related to extensional faults, and produce mud mounds $\sim 0.4$ to $2 \mathrm{~km}$ wide and $\sim 20-200 \mathrm{~m}$ high. Along the Seamount and Cocos Ridge segments, fluid seeps are associated with normal faults and fractures associated with upper plate uplift and slide scars caused by subducting seafloor relief. Sahling et al. [2008] speculate that perhaps two to three times more seeps exist than have been mapped. Examples of focused seeps with clear thermal signatures include Mound Culebra [Grevemeyer et al., 2004] just north of seismic line BGR99-39 (Figures 2a and 5), Mound
11 associated with seismic line BGR99-17 (Figure 8), seeps associated with the slump deposit and headwall along line SO81-13 (Figure 7), and the seep just off seismic line SO81-3 (Figures 2 and 7). Heat flow anomalies associated with these seeps can be quite large relative to background heat flow. Temperature-depth profiles associated with these seeps are linear suggesting flow rates of less than $1 \mathrm{~cm} \mathrm{yr}^{-1}$ [Grevemeyer et al., 2004; Ranero et al., 2008]. Modeling of pore water chemistry collected from gravity cores at seeps indicates flow rates across most of the structure are 0.1 to $0.3 \mathrm{~cm} / \mathrm{yr}$ [Hensen et al., 2004; Ranero et al., 2008]. Other manifestations of advective fluid flow through the margin may be gaps in BSRs associated with the presence of seeps (SO81-9, Figure 6; SO81-2 and SO81-3, Figure 7) and the high-wave number heat flow anomalies $12-22 \mathrm{~km}$ landward of the deformation front on observed on seismic line SO81-21 (Figure 6).

[43] We provide an estimate of the flow rate needed to generate these anomalies by solving the onedimensional steady state advection diffusion equation,

$$
\frac{\partial^{2} T}{\partial z^{2}}-\frac{\theta \rho_{w} c_{w}}{k_{e}} V_{z} \frac{\partial T}{\partial z}=0
$$

where $z$ is depth, $T$ is temperature, $\theta$ is porosity, $k_{e}$ is effective thermal conductivity, $\rho_{\mathrm{w}} \mathrm{c}_{\mathrm{w}}$ is the heat capacity of water and $V_{z}$ is the mean vertical velocity. At the seafloor we use a constant temperature boundary, $T_{o}$, and a constant gradient, $\Gamma_{\mathrm{L}}$, at depth, $L$. The solution for this model is,

$$
T(z)=T_{o}+\frac{\Gamma_{L} L}{\beta} \frac{\exp \left(\frac{\beta z}{L}\right)-1}{\exp (\beta)},
$$

where $\beta$ is the Peclet number,

$$
\beta=\frac{\theta \rho_{w} c_{w} V_{z} L}{k_{e}} .
$$

At $z=0$, heat flow can be expressed as,

$$
q=\frac{k_{e} \Gamma_{L}}{\exp (\beta)} .
$$

In this calculation we assume that the depth of the decollement is $2 \mathrm{~km}$ as interpreted from the seismic image (Figure 2), the porosity is $20 \%$ and that the background heat flux is $50 \mathrm{~mW} \mathrm{~m}$. With the exception of line SO81-2 (Figure 7) probe values of heat flow are generally 20 to $60 \mathrm{~mW} \mathrm{~m}^{-2}$ larger than BSR-derived estimates at slumps. These heat flow anomalies are consistent with flow rates of 7 to $15 \mathrm{~mm} \mathrm{yr}^{-1}$. 
[44] Landslides and slumps can also produce variability in heat flow transects and it is interesting to consider the potential effect of a landslide or slump on heat flow. The clearest example of an erosive event is along seismic line SO81-13 (Figures 2d and 7) where a slump has removed approximately $750 \mathrm{~m}$ of material. This appears to be a relatively recent event because a BSR has not reformed within the downslope slump material. If we assume that the slump occurred instantaneously, and if the background thermal gradient is about $50^{\circ} \mathrm{C} / \mathrm{km}$ then the top of the unroofed material would have a temperature of about $38^{\circ} \mathrm{C}$ just prior to unroofing. Instantaneous unroofing increases the near subbottom heat flow. The change in heat flow, $\Delta \mathrm{q}$, due to a step change in temperature, $\Delta \mathrm{T}$, can be expressed as,

$$
\Delta q=k_{e} \frac{\Delta T}{\sqrt{\pi \alpha t}} \exp \left(\frac{-z^{2}}{4 \alpha t}\right),
$$

where $\alpha$ is thermal diffusivity, and $t$ is time. At $z=0$, the change in heat flow scales with the inverse square root of time. To produce the observed heat flow anomaly of approximately $100 \mathrm{~mW} \mathrm{~m}^{-2}$, equation (8) requires that the slump occurred within about 1000 years before present, assuming a thermal diffusivity of $32 \mathrm{~km}^{2} \mathrm{Ma}^{-1}$ characteristic of rocks.

[45] Heat flow data within about $10 \mathrm{~km}$ of the deformation front shows relatively large variability (BGR99-44, Figure 5; SO81-8 and SO81-21, Figure 6; SO81-2, Figure 7; P-1600, Figure 8). This data is associated with the sedimentary frontal prism and the high wave number variability may be associated with lateral changes in the intensity of faulting during kneading of sediment, or fracturing due to deformation caused by relative low-relief subducting topography like horst and graben structures [e.g., Zwart et al., 1996; Ganguly et al., 2000].

[46] Two other heat flow features are important to note. The first is the heat flow high along seismic line SO81-5 that is remarkable in its amplitude, width, and good agreement between probe and BSR values of heat flow (Figures $2 \mathrm{e}$ and 8). A series of distinct nearly horizontal reflections depict a subducted horst associated located under this heat flow anomaly and may indicate rapid fluid flow contained within the subducting oceanic crust such that heat transfer between the BSR and the seafloor is dominantly conductive. Fluid flow through the subducting oceanic crust is modeled and discussed by Harris et al. [2010]. The second feature is the extremely high frontal heat flow values associated with seismic lines BGR99-15 and P-1600 (Figures 8d and 8e). Both seismic lines are in the southern region of the Cocos Ridge segment where the buoyancy and seafloor relief of the Cocos Ridge has modified the margin so that it is short and steep. Almost no seeps have been identified in this area and it appears that fluid generated from both compaction and higher neartoe temperatures causing shallow dehydration [Ranero et al., 2008] may be driving intense fluid flow along the decollement and frontal prism faults in the region. This fluid flow may help explain the anomalously high heat flow and rapid decrease in heat flow associated with the transition from the deformation front to the rock body of the margin.

\section{Conclusions}

[47] The combination of in situ measurements and BSR estimates of heat flow along the Costa Rican margin provides insight into the thermal regime of the margin. Our analysis leads us to the following conclusions:

[48] 1. There is an excellent correlation between in situ values of heat flow and those derived from the position of BSRs compared to accretionary prisms. This allows the conclusion that heat transfer between the BSR and seafloor is dominantly conductive.

[49] 2. Heat flow variations along strike are significant and likely reflect the thermal state of the subducting Cocos Plate. In general the margin is relatively cool offshore the northern Nicoya Peninsula where EPR generated oceanic crust is subducting. South of the plate suture the margin is relatively warm and heat flow near the deformation front increases to the south.

[50] 3. High-wave number $(5-10 \mathrm{~km})$ heat flow variation along transects is significant. This variation is greater south of the plate suture and is spatially associated with the subduction of basement relief and is likely due to advective fluid flow through fractures related to seamount subduction and normal faults related to upper plate extension. In some cases high heat flow may be associated with the rapid removal of seafloor material during slumping.

\section{Acknowledgments}

[51] This research was support by an NSF award (OCE0637120) to R.N.H. We thank R. von Huene, P. Fulton, and G. Spinelli for helpful comments. Heat flow data acquisition 
was funded by the German Science Foundation (DFG) through grant Vi 133/7-1 to H.V. and I.G. and the SFB 574 "Volatiles and fluids in subduction zones" at Christan-Albrechts University, Kiel. This is a contribution of the Barcelona Center for Subsurface Imaging (Barcelona-CSI) supported by the Kaleidoscope project of REPSOL.

\section{References}

Barckhausen, U., C. R. Ranero, R. von Huene, S. C. Cande, and H. A. Roeser (2001), Revised tectonic boundaries in the Cocos Plate off Costa Rica: Implications for the segmentation of the convergent margin and for plate tectonic models, J. Geophys. Res., 106, 19,207-19,220, doi:10.1029/ 2001JB000238.

Blackwell, D. D., J. L. Steele, and C. A. Brott (1980), The terrain effect on terrestrial heat flow, J. Geophys. Res., 85, 4757-4772, doi:10.1029/JB085iB09p04757.

Brown, K. M., and N. L. Bangs (1995), Thermal regime of the Chile triple junction: Constraints provided by downhole temperature measurements and distribution of gas hydrate, Proc. Ocean Drill. Program Initial Rep., 14, 259-275.

Chan, L.-H., and M. Kastner (2000), Lithium isotopic compositions of pore fluids and sediments in the Costa Rica subduction zone: Implications for fluid processes and sediment contribution to the arc volcanoes, Earth Planet. Sci. Lett., 183, 275-290, doi:10.1016/S0012-821X(00)00275-2.

Christeson, G. L., K. D. McIntosh, T. H. Shipley, E. R. Flueh, and H. Goedde (1999), Structure of the Costa Rica convergent margin, offshore Nicoya Peninsula, J. Geophys. Res., 104, 25,443-25,468, doi:10.1029/1999JB900251.

Davis, E. E., and H. Villinger (2006), Transient formation fluid pressures and temperatures in the Costa Rica forearc prism and subducting oceanic basement: CORK monitoring at ODP Sites 1253 and 1255, Earth Planet. Sci. Lett., 245, 232-244, doi:10.1016/j.epsl.2006.02.042.

Davis, E. E., R. D. Hyndman, and H. Villinger (1990), Rates of fluid expulsion across the northern Cascadia accretionary prism: Constraints from new heat flow and multichannel seismic reflection data, J. Geophys. Res., 95, 8869-8889, doi:10.1029/JB095iB06p08869.

DeMets, C. (2001), A new estimate for present-day CocosCaribbean plate motion: Implications for slip along the Central American volcanic arc, Geophys. Res. Lett., 28, 4043-4046, doi:10.1029/2001GL013518.

Dickens, G. R., and M. S. Quinby-Hunt (1994), Methane hydrate stability in seawater, Geophys. Res. Lett., 21, 2115-2118, doi:10.1029/94GL01858.

Fisher, A. T., C. A. Stein, R. N. Harris, K. Wang, E. A. Silver, M. Pfender, M. Hutnak, A. Cherkaoui, R. Bodzin, and H. Villinger (2003), Abrupt thermal transition reveals hydrothermal boundary and role of seamounts within the Cocos Plate, Geophys. Res. Lett., 30(11), 1550, doi:10.1029/ 2002 GL016766.

Ganguly, N., G. D. Spence, N. R. Chapman, and R. D. Hyndman (2000), Heat flow variations from bottom simulating reflectors on the Cascadia margin, Mar. Geol., 164, 53-68, doi:10.1016/S0025-3227(99)00126-7.

Gardner, T. W., et al. (2001), Holocene fore arc block rotation in response to seamount subduction, southeastern Peninsula de Nicoya, Costa Rica, Geology, 29, 151-154, doi:10.1130/ 0091-7613(2001)029<0151:HFBRIR >2.0.CO;2.

Grevemeyer, I., and H. Villinger (2001), Gas hydrate stability and the assessment of heat flow through continental margins,
Geophys. J. Int., 145, 647-660, doi:10.1046/j.0956-540x. 2001.01404.x.

Grevemeyer, I., A. Rosenberger, and H. Villinger (2000), Natural gas hydrates on the continental slope off Pakistan: Constraints from seismic techniques, Geophys. J. Int., 140, 295-310, doi:10.1046/j.1365-246x.2000.00009.x.

Grevemeyer, I., et al. (2004), Fluid flow through active mud dome Mound Culebra offshore Nicoya Peninsula, Costa Rica: Evidence from heat flow surveying, Mar. Geol., 207, 145-157, doi:10.1016/j.margeo.2004.04.002.

Harris, R. N., G. Spinelli, C. R. Ranero, I. Grevemeyer, H. Villinger, and U. Barckhausen (2010), Thermal regime of the Costa Rican convergent margin: 2. Thermal models of the shallow Middle America subduction zone offshore Costa Rica, Geochem. Geophys. Geosyst., doi:10.1029/2010GC003273, in press.

Hartmann, A., and H. Villinger (2002), Inversion of marine heat flow measurements by expansion of the temperature decay function, Geophys. J. Int., 148, 628-636, doi:10.1046/ j.1365-246X.2002.01600.x.

Hensen, C., and K. Wallmann (2005), Methane formation at Costa Rica continental margin: Constraints for gas hydrate inventory and cross-décollement fluid flow, Earth Planet. Sci. Lett., 236, 41-60, doi:10.1016/j.eps1.2005.06.007.

Hensen, C., K. Wallmann, M. Schmidt, C. R. Ranero, and E. Suess (2004), Fluid expulsion related to mud extrusion off Costa Rica-A window to the subducting slab, Geology, 32, 201-204, doi:10.1130/G20119.1.

Hey, R. N. (1977), Tectonic evolution of the Cocos-Nazca spreading center, Geol. Soc. Am. Bull., 88, 1404-1420, doi:10.1130/0016-7606(1977)88<1404:TEOTCS $>2.0 . C O ; 2$.

Hinz, K., R. von Huene, and C. R. Ranero (1996), Tectonic structure of the convergent Pacific margin offshore Costa Rica from multichannel seismic reflection data, Tectonics, 15, 54-66, doi:10.1029/95TC02355.

Hutnak, M., et al. (2007), The thermal state of 18-24 Ma upper lithosphere subducting below the Nicoya Peninsula, northern Costa Rica margin, in The Seismogenic Zone of Subduction Thrust Faults, edited by T. Dixon and C. Moore, pp. 42-85, Columbia Univ. Press, New York.

Hutnak, M., A. T. Fisher, R. Harris, C. Stein, K. Wang, G. Spinelli, M. Schindler, H. Villinger, and E. Silver (2008), Surprisingly large heat and fluid fluxes driven through midplate outcrops on ocean crust, Nat. Geosci., 1, 611-614, doi:10.1038/ngeo264.

Hyndman, R. D., E. E. Davis, and J. A. Wright (1979), The measurement of marine geothermal heat flow by a multipenetration probe with digital acoustic telemetry and in situ thermal conductivity, Mar. Geophys. Res., 4, 181-205, doi:10.1007/BF00286404.

Hyndman, R. D., J. P. Foucher, M. Yamano, A. T. Fisher, and the ODP Leg 131 Scientific Party (1992), Deep sea bottom simulating reflectors: Calibration of the base of the hydrate stability field as used for heat flow estimates, Earth Planet. Sci. Lett., 109, 289-301, doi:10.1016/0012-821X(92)90093-B.

Kaul, N., A. Rosenberger, and H. Villinger (2000), Comparison of measured and BSR-derived heat flow values, Makran accretionary prism, Pakistan, Mar. Geol., 164, 37-51, doi:10.1016/S0025-3227(99)00125-5.

Kimura, G., et al. (1997), Proceedings of the Ocean Drilling Program, Initial Reports, vol. 170, doi:10.2973/odp.proc. ir.170.1997, Ocean Drill. Program, College Station, Tex.

Langseth, M. G., and E. A. Silver (1996), The Nicoya Convergent Margin - A region of exceptionally low heat flow, Geophys. Res. Lett., 23(8), 891-894, doi:10.1029/96GL00733. 
Lister, C. R. B. (1979), The pulse-probe method of conductivity measurement, Geophys. J. R. Astron. Soc., 57, 451-461, doi:10.1111/j.1365-246X.1979.tb04788.x.

McAdoo, B. G., et al. (1996), Seafloor structural observations, Costa Rica accretionary prism, Geophys. Res. Lett., 23, 883-886, doi:10.1029/96GL00731.

Minshull, T. A., R. Barolome, S. Byrne, and J. Danobeitia (2005), Low heat flow from young oceanic lithosphere at the Middle America Trench off Mexico, Earth Planet. Sci. Lett., 239, 33-41, doi:10.1016/j.epsl.2005.05.045.

Morris, J. D., et al. (2003), Proceedings of the Ocean Drilling Program, Initial Reports, vol. 205, doi:10.2973/odp.proc. ir.205.2003, Ocean Drill. Program, College Station, Tex.

Müller, C., C. Bönnemann, and S. Neben (2007), AVO study of a gas-hydrate deposit, offshore Costa Rica, Geophys. Prospect., 55, 719-735, doi:10.1111/j.1365-2478.2007.00632.x.

Pecher, I. A., C. R. Ranero, R. von Huene, T. A. Minshull, and S. C. Singh (1998), The nature and distribution of bottom simulating reflectors at the Costa Rican convergent margin, Geophys. J. Int., 133, 219-229, doi:10.1046/j.1365-246X.1998. 00472.x.

Pecher, I. A., N. Kukowski, C. R. Ranero, and R. von Huene (2001), Gas hydrates along the Peru and Middle America trench systems, in Natural Gas Hydrates: Occurrence, Distribution, and Dynamics, Geophys. Monogr. Ser., vol. 124, edited by C. K. Paull and W. P. Dillon, pp. 257-271, AGU, Washington, D. C.

Pfender, M., and H. Villinger (2002), Miniaturized data loggers for deep sea sediment temperature gradient measurements, Mar. Geol., 186, 557-570, doi:10.1016/S0025-3227(02) 00213-X

Ranero, C. R., and R. von Huene (2000), Subduction erosion along the Middle America convergent margin, Nature, 404, 748-752, doi:10.1038/35008046.

Ranero, C. R., R. von Huene, E. Flueh, M. Duarte, D. Baca, and K. McIntosh (2000), A cross section of the convergent Pacific margin of Nicaragua, Tectonics, 19, 335-357, doi:10.1029/1999TC900045.

Ranero, C. R., R. von Huene, W. Weinrebe, and U. Barckhausen (2007), Convergent margin tectonics of Middle America: A marine perspective, in Central America: Geology, Resources and Hazards, edited by J. Bunschuh and G. E. Alvarado, pp. 239-265, Taylor and Francis, London.

Ranero, C. R., I. Grevemeyer, H. Sahling, U. Barchkhausen, C. Hensen, K. Wallmann, W. Weinrebe, P. Vannucchi, R. von Huene, and K. McIntosh (2008), Hydrogeological system of erosional convergent margins and its influence on tectonics and interplate seismogenesis, Geochem. Geophys. Geosyst., 9, Q03S04, doi:10.1029/2007GC001679.

Ruppel, C. (1997), Anomalously cold temperatures observed at the base of the gas hydrate stability zone, U.S. Atlantic passive margin, Geology, 25, 699-702, doi:10.1130/00917613(1997)025<0699:ACTOAT>2.3.CO;2.

Ruppel, C., and M. Kinoshita (2000), Fluid, methane, and energy flux in an active margin gas hydrate province, offshore Costa Rica, Earth Planet. Sci. Lett., 179, 153-165, doi:10.1016/S0012-821X(00)00096-0.

Saffer, D. M., E. A. Silver, A. T. Fisher, H. Tobin, and K. Moran (2000), Inferred pore pressures at the Costa Rica subduction zone: Implications for dewatering processes, Earth Planet. Sci. Lett., 177, 193-207, doi:10.1016/S0012821X(00)00048-0.

Sahling, H., D. G. Masson, C. R. Ranero, V. Hühnerbach, W. Weinrebe, I. Klaucke, D. Bürk, W. Brückmann, and
E. Suess (2008), Fluid seepage at the continental margin offshore Costa Rica and southern Nicaragua, Geochem. Geophys. Geosyst., 9, Q05S05, doi:10.1029/2008GC001978.

Schmidt, M. C., M. Schmidt, C. Hensen, T. Morz, C. Muller, I. Grevemeyer, K. Wallmann, S. Mau, and N. Kaul (2005), Methane hydrate accumulation in "Mound 11" mud volcano, Costa Rica forearc, Mar. Geol., 216, 83-100, doi:10.1016/j.margeo.2005.01.001.

Screaton, E. J., and D. M. Saffer (2005), Fluid expulsion and overpressure development during initial subduction at the Costa Rica convergent margin, Earth Planet. Sci. Lett., 233, 361-374, doi:10.1016/j.eps1.2005.02.017.

Shipley, T. H., M. H. Houston, R. T. Buffler, F. J. Shaub, K. J. McMillen, J. W. Ladd, and J. L. Worzel (1979), Seismic evidence for the widespread occurrence of possible gas-hydrate horizons on continental slopes and rises, Am. Assoc. Pet. Geol. Bull., 63, 2204-2213.

Shipley, T. H., K. D. McIntosh, E. A. Silver, and P. L. Stoffa (1992), Three-dimensional seismic imaging of the Costa Rica accretionary prism: Structural diversity in a small volume of the lower slope, J. Geophys. Res., 97, 4439-4459, doi:10.1029/91JB02999.

Silver, E., M. Kastner, A. Fisher, J. Morris, K. McIntosh, and D. Saffer (2000), Fluid flow paths in the Middle America Trench and Costa Rica margin, Geology, 28, 679-682, doi:10.1130/0091-7613(2000)28<679:FFPITM>2.0.CO;2.

Tucholke, B. E., G. M. Bryan, and J. I. Ewing (1977), Gashydrate horizons detected in seismic-profiler data from the western North Atlantic, Am. Assoc. Pet. Geol. Bull., 61, 698-707.

Vannucchi, P., C. R. Ranero, S. Galeotti, S. M. Straub, D. W. Scholl, and K. McDougall-Ried (2003), Fast rates of subduction erosion along the Costa Rica Pacific margin: Implications for nonsteady rates of crustal recycling at subduction zones, J. Geophys. Res., 108(B11), 2511, doi:10.1029/ 2002JB002207.

von Huene, R., et al. (1985), Site 565, Initial Rep. Deep Sea Drill. Program, 84, 21-78, doi:10.2973/dsdp.proc.84.1985

von Huene, R., C. R. Ranero, W. Weinrebe, and K. Hinz (2000), Quaternary convergent margin tectonics of Costa Rica, segmentation of the Cocos Plate, and Central American volcanism, Tectonics, 19, 314-334, doi:10.1029/1999TC001143.

von Huene, R., C. R. Ranero, and P. Watts (2004), Tsunamigenic slope failure along the Middle America Trench in two tectonic settings, Mar. Geol., 203, 303-317, doi:10.1016/ S0025-3227(03)00312-8.

Walther, C. H. E. (2003), The crustal structure of the Cocos ridge off Costa Rica, J. Geophys. Res., 108(B3), 2136, doi:10.1029/2001JB000888.

Wang, K., and E. E. Davis (1992), Thermal effects of marine sedimentation in hydrothermally active areas, Geophys. J. Int., 110, 70-78, doi:10.1111/j.1365-246X.1992.tb00714.x.

Yamano, M., S. Uyeda, Y. Aoki, and T. H. Shipley (1982), Estimates of heat flow derived from gas hydrates, Geology, 10, 339-343, doi:10.1130/0091-7613(1982)10<339:EOHFDF> 2.0.CO;2.

Ye, S., J. Bialas, E. R. Flueh, A. Stavenhagen, and R. von Huene (1996), Crustal structure of the Middle American Trench off Costa Rica from wide-angle seismic data, Tectonics, 15, 1006-1021, doi:10.1016/0012-821X(95)00244-7

Zwart, G., J. C. Moore, and G. R. Cochrane (1996), Variations in temperature gradients identify active faults in the Oregon accretionary prism, Earth Planet. Sci. Lett., 139, 485-495, doi:10.1016/0012-821X(95)00244-7. 